\title{
A 3D finite element model for the vibration analysis of asymmetric rotating machines
}

\author{
A. Lazarus ${ }^{\text {a }}$, B. Prabel ${ }^{\text {b,* }}$, D. Combescure ${ }^{\text {b }}$ \\ a Laboratoire de Mecanique des Solides, Ecole Polytechnique, 91128 Palaiseau Cedex, France \\ ${ }^{\text {b } C E A, ~ D E N, ~ D M 2 S, ~ S E M T, ~ D Y N, ~ F-91191 ~ G i f-s u r-Y v e t t e, ~ F r a n c e ~}$
}

This paper suggests a 3D finite element method based on the modal theory in order to analyse linear periodically time-varying systems. Presentation of the method is given through the particular case of asymmetric rotating machines. First, Hill governing equations of asymmetric rotating oscillators with two degrees of freedom are investigated. These differential equations with periodic coefficients are solved with classic Floquet theory leading to parametric quasimodes. These mathematical entities are found to have the same fundamental properties as classic eigenmodes, but contain several harmonics possibly responsible for parametric instabilities. Extension to the vibration analysis (stability, frequency spectrum) of asymmetric rotating machines with multiple degrees of freedom is achieved with a fully 3D finite element model including stator and rotor coupling. Due to Hill expansion, the usual degrees of freedom are duplicated and associated with the relevant harmonic of the Floquet solutions in the frequency domain. Parametric quasimodes as well as steady-state response of the whole system are ingeniously computed with a component-mode synthesis method. Finally, experimental investigations are performed on a test rig composed of an asymmetric rotor running on nonisotropic supports. Numerical and experimental results are compared to highlight the potential of the numerical method.

\section{Introduction}

Understanding physics of rotating machines is essential in many application fields. For example, safety and service life in power plants rotating machinery such as turbo-alternator [1] or GT-MHR reactor project [2] are directly linked to a highquality control of their vibratory behaviour. Basic rotordynamics modelling for design and predictive maintenance [3] is usually sufficient to solve most of the industrial problems encountered but there is still advanced topics to investigate [4]. One of them concerns the 3D global modelling of asymmetric rotating systems.

Behaviour of unsymmetrical large rotating machines includes here a lot of cases of practical importance such as dynamics of rotors with shape imperfections [5], vibratory signature of cracked rotors for on-line monitoring [6,1], lateral instabilities of drill-strings [7]. Under the assumption of constant rotating speed, all these problems have in common to be linear periodically time-varying systems [4], i.e. governed by differential equations with periodic coefficients. Solutions of these equations are generally determined thanks to the well-known Floquet theory [8]. These solutions can be computed in the time domain by direct integration of the Floquet transition matrix as in [9]. An other possibility is to determine these

\footnotetext{
* Corresponding author.

E-mail addresses: lazarus@ladhyx.polytechnique.fr (A. Lazarus), benoit.prabel@cea.fr (B. Prabel), didier.combescure@f4e.europa.eu (D. Combescure).
} 
solutions in the frequency domain by analysing the set of equivalent linear time-invariant equations obtained by the Hill expansion [10].

First modelling of unsymmetrical rotating machines were performed using simple rotating oscillators with anisotropic stiffnesses [5]. Indeed, for these academic models, Hill governing equations are easily established and solved [11]. Extension to the finite element models in stationary coordinates is referred in [12] for unsymmetrical rotors and in [4,13] for both anisotropic rotors and supports. Still in the inertial frame, a modal analysis for periodically time-varying rotors is suggested in [6,14] and allows reasonable computation time for simple rotor models (with shape imperfection, transverse cracks). However, the standard representation in the inertial frame limits our investigations to rotating shafts modelling with beam elements [2]. A complete 3D modelling of rotors described in the rotating frame [4] enables to capture a richer kinematics (blades, flexible discs, original shapes, etc.) and facilitates the dynamic studies of asymmetric rotors [15].

This paper focuses on the global vibratory behaviour of asymmetric rotating machines taking into account the rotorstator interactions. It is inspired by the 3D finite element model developed in [2] for axisymmetrical rotating machines. More generally, it suggests a 3D finite element method based on modal analysis to solve linear periodically time-varying systems.

In Sections 2-4, the dynamic behaviour of asymmetric rotating machines is investigated through oscillators with two degrees of freedom written in complex coordinates. Indeed, oscillators with anisotropic stiffnesses in both rotating and inertial frame are useful for an introductory study of rotors with open cracks or shape imperfections. We explain the numerical method used to compute the solution of the non-autonomous governing equations. By considering Floquet solutions and Hill's infinite determinant, it is found that the dynamics of these rotating oscillators can be intrinsically defined by eigenmodes in the same way as in the autonomous case. As a consequence, free whirling and forced response are a linear combination of these parametric quasimodes. According to the linearity assumption, stability of the Floquet solution is determined by the stability of the parametric quasimodes, i.e. their decay rates.

Section 5 concerns the extension of the previous concepts to the multiple degrees of freedom governing equation arising from the 3D finite element discretization of a rotating machine with a possible shape imperfection. Due to the Hill expansion of the degrees of freedom in the frequency domain, the parametric quasimodes are computed with the modal synthesis method. The boundary conditions between substructures model the constant rotational speed $\Omega$ and the rotorstator interactions. The last part takes advantage of the numerical tools previously introduced for the dynamic investigation of an academic test rig composed of a rectangular vertical rotor running on anisotropic supports. The comparison between numerical and experimental results gives us a first insight of the efficiency of the proposed method.

\section{Academic rotating oscillator with asymmetric stiffnesses}

\subsection{Influence of imperfections on the dynamic behaviour of rotating systems}

The flexural behaviour of an horizontal shaft supported by anisotropic supports is represented in Fig. 1(a) [3]. The gyroscopic coupling due to possible rotating discs is neglected to focus only on the influence of imperfections. Thanks to a beam element model representing the position of the rotating shaft in an inertial frame $\mathcal{R}$, a classic modal reduction can be used for stability analysis and prediction of the global dynamics [4]. The representation in the modal basis is given in Fig. 1(b) when keeping the two first flexural eigenmodes which are sufficient for qualitative results.

(a)

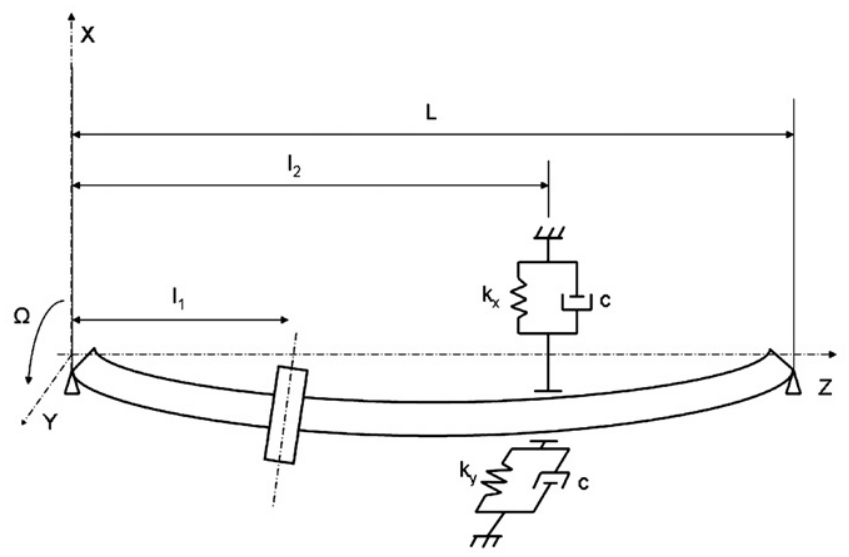

(b)

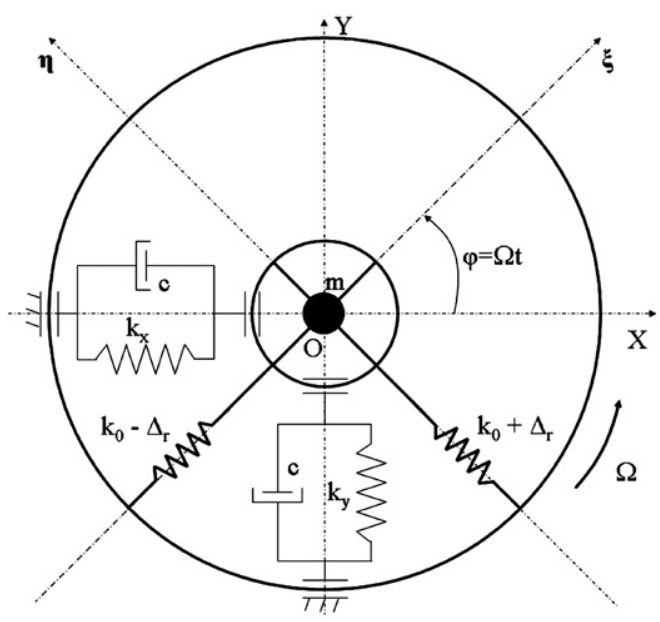

Fig. 1. Asymmetric shaft on nonisotropic bearings: (a) first beam mode in the $x$-direction; (b) representation in the modal basis. 
While the modal rotating stiffness $k_{0}$ is isotropic in the rotating frame $\mathcal{R}^{\prime}$ in the case of healthy shafts (basic rotordynamics), shape imperfections or open cracks [16] yield to an anisotropic stiffness in $R^{\prime}$. The latter is characterized by the added stiffness $\Delta_{r}$ directly linked to the crack depth or shape asymmetry (Fig. 1(b)). Under the reasonable assumptions of small displacements around the static equilibrium position and constant rotational speed $\Omega$, the linear periodically timevarying system written in stationary generalized coordinates $(x(t), y(t))$ is obtained [3]

$$
\left\{\begin{array}{l}
m \ddot{x}+c \dot{x}+\left(k_{0}+k_{x}\right) x-\Delta_{r} \cos 2 \Omega t x-\Delta_{r} \sin 2 \Omega t y=0 \\
m \ddot{y}+c \dot{y}+\left(k_{0}+k_{y}\right) x-\Delta_{r} \sin 2 \Omega t x+\Delta_{r} \cos 2 \Omega t y=0
\end{array}\right.
$$

where $m$ is the rotor modal mass, $c$ is the modal viscous damping and $k_{x}, k_{y}$ are, respectively, the modal fixed stiffness in the $x$ - and $y$-directions.

\subsection{Governing equations in complex coordinates}

In the case of rotating systems, transverse displacements are generally coupled and the use of complex coordinates allows us to greatly simplify the governing equations of models [12]. By introducing $z=x+$ iy the generalized complex stationary coordinate and $\bar{z}$ its conjugate, Eq. (1) becomes

$$
m \ddot{z}(t)+c \dot{z}(t)+\delta z(t)+\varepsilon \bar{z}(t)+k_{r}(t) \bar{z}(t)=0
$$

where $\delta=\left(k_{x}+k_{y}\right) / 2+k_{0}$ is the mean stiffness and $\varepsilon=\left(k_{y}-k_{x}\right) / 2$ is the fixed stiffness deviation. For $\Omega>0$, the rotor imperfection $\Delta_{r}$ introduces a periodic coefficient $k_{r}(t)$ in the differential equation (2). In our case, $k_{r}(t)$ is a harmonic operator with a period $T=\pi / \Omega$ (fundamental frequency $2 \Omega$ ) and reads simply

$$
k_{r}(t)=-\Delta_{r} \mathrm{e}^{\mathrm{i} 2 \Omega t}
$$

The governing equation (2) belongs to homogeneous differential equations with periodic coefficients. In this paper, this typical Hill equation is studied in the frequency domain well adapted to linear rotordynamics considering constant rotational speed. Moreover, this choice is necessary when considering the computational time for finite element modelling.

\subsection{Numerical data}

This paragraph specifies the numerical data used for the further numerical computations of Eq. (2). In the modal basis, the rotating oscillator is modelled by $n=2$ degrees of freedom. The natural frequencies at standstill $(\Omega=0)$ in the $x$ and $y$ directions are naturally defined by the following expressions:

$$
\omega_{0}^{x}=\sqrt{\frac{\delta-\varepsilon-\Delta_{r}}{m}} \text { and } \omega_{0}^{y}=\sqrt{\frac{\delta+\varepsilon+\Delta_{r}}{m}}
$$

We introduce also the dimensionless fixed stiffness anisotropy ratio $\alpha_{n}=k_{x} / k_{y}$ and the rotating imperfection ratio $\alpha_{r}=\left(k_{0}-\Delta_{r}\right) /\left(k_{0}+\Delta_{r}\right)$. In the following, the academic data are chosen, so that $\omega_{0}^{x}=1 \mathrm{rad} / \mathrm{s}$. The dimensionless damping $\zeta$ is then defined relatively to $\omega_{0}^{x}$ according to $\zeta=c \omega_{0}^{x} / 2\left(\delta-\varepsilon-\Delta_{r}\right)$. To highlight the influence of imperfections, we consider important fixed and rotating stiffness ratios $\alpha_{n}=3$ and $\alpha_{r}=2$.

\section{Analysis of Hill's equation introducing parametric quasimodes}

\subsection{Floquet theory and Hill's method}

Thanks to the Floquet theory [8], the solution of the linear differential equation (2) can be written as the sum of $n=2$ independent solutions

$$
z(t)=Z_{D}(t) \mathrm{e}^{\mathrm{i} \alpha t}+Z_{R}(t) \mathrm{e}^{-\mathrm{i} \bar{\alpha} t}
$$

where both $Z_{D}(t)$ and $Z_{R}(t)$ are $T$-periodic functions of time with the same period as the coefficient $k_{r}(t)$ and $\alpha$ is the fundamental complex frequency and $\bar{\alpha}$ its conjugate. Unknown periodic functions $Z_{D}$ and $Z_{R}$ can be expressed by the general Fourier series

$$
Z(t)=\sum_{j=-\infty}^{j=+\infty} Z_{j} \mathrm{e}^{\mathrm{i} j(2 \pi / T) t}
$$

where $Z_{j}$ is the harmonic contribution for the $j$ th harmonic. The periodic stiffness $k_{r}(t)$ is imposed by the physical situation and can be expanded as well in the convergent trigonometric series

$$
k_{r}(t) \approx \sum_{s=-S}^{s=+S} \Delta_{s} \mathrm{e}^{\mathrm{i} s(2 \pi / T) t}
$$


where $\Delta_{s}$ is the $s$ th stiffness Fourier coefficient. In our asymmetric case, the rotating stiffness $k_{r}(t)$ is harmonic with a period $T=\pi / \Omega$. Taking $T$ into account in expression (6), the solution (5) can be expressed as the infinite sum of relevant harmonics

$$
z(t)=\sum_{j=-\infty}^{j=+\infty} Z_{D j} \mathrm{e}^{\mathrm{i}(\alpha+2 j \Omega) t}+\sum_{j=-\infty}^{j=+\infty} Z_{R j} \mathrm{e}^{-\mathrm{i}(\bar{\alpha}+2 j \Omega) t}
$$

By replacing, respectively, $k_{r}(t)$ and $z(t)$ by their expressions (3) and (8) in Eq. (2) and by separately equating to zero each harmonic, we obtain the infinite set of algebraic equations

$$
\left[\begin{array}{cccccccc}
- & - & - & - & - & - & - & - \\
- & A_{-1} & \varepsilon & 0 & 0 & 0 & 0 & - \\
- & \varepsilon & A_{-1} & -\Delta_{r} & 0 & 0 & 0 & - \\
- & 0 & -\Delta_{r} & A_{0} & \varepsilon & 0 & 0 & - \\
- & 0 & 0 & \varepsilon & A_{0} & -\Delta_{r} & 0 & - \\
- & 0 & 0 & 0 & -\Delta_{r} & A_{1} & \varepsilon & - \\
- & 0 & 0 & 0 & 0 & \varepsilon & A_{1} & - \\
- & - & - & - & - & - & - & -
\end{array}\right]\left\{\begin{array}{c}
- \\
Z_{D-1} \\
\bar{Z}_{R-1} \\
Z_{D 0} \\
\bar{Z}_{R 0} \\
Z_{D 1} \\
\bar{Z}_{R 1} \\
-
\end{array}\right\} \approx\{0\}
$$

where $A_{j}=\delta-m(\alpha+2 j \Omega)^{2}+\mathrm{i} c(\alpha+2 j \Omega)$. This eigenproblem in $\alpha$ results of non-autonomous equations (2) expressed in the frequency domain for a given $\Omega$. Thus, rotor imperfections introduce more or less coupling between harmonics of the periodic solution expressed in the fixed frame $\mathcal{R}$. Due to the infinite expansion (6), the determinant associated with (9) is called the Hill infinite determinant [10].

\subsection{Concept of parametric quasimodes}

Let $\operatorname{det}_{j_{\max }}$ be the $j_{\max }$ order determinant associated with the eigenproblem (9) and $a_{j p}$ the coefficients of the $j$ th row and $p$ th column of the matrix in (9). According to [17], det $j_{\max }$ converges, i.e. tends to a finite and determined limit det, if the series $\sum_{j}\left|a_{j p} / a_{j j}\right|$ with $p \neq j$ converges and it is always the case for Hill determinant.

By solving eigenproblem (9) in the state space truncated to the $j_{\max }$ harmonic order for a given $\Omega$, one obtains $2 n \times\left(2 j_{\max }+1\right)$ eigenvalues $\alpha_{l}$ where $n=2$ is the number of degrees of freedom. According to (8), each eigenvalues is associated with a complex eigenvector $\Phi_{l}=\left[Z_{D-j_{\max }} \ldots Z_{R j_{\max }}\right]$ expressed in the time domain as

$$
\Phi_{l}(t)=\sum_{-j_{\max }}^{+j_{\max }} Z_{D j l} \mathrm{e}^{\mathrm{i}\left(\alpha_{l}+2 j \Omega\right) t}+\sum_{-j_{\max }}^{+j_{\max }} Z_{R j l} \mathrm{e}^{-\mathrm{i}\left(\bar{\alpha}_{l}+2 j \Omega\right) t}
$$

where $Z_{D j l}$ and $Z_{R j l}$ are the $j$ th harmonic contributions of the $l$ th eigenvector.

According to Poincare's convergence of Hill infinite determinant, as $j_{\max }$ infinitely increases, the set of eigenvalues tends to $n$ independent families in which

$$
\alpha_{l}^{k}= \pm\left(\omega^{k}+2 l \Omega\right)
$$

where $l \in\left[-j_{\max },+j_{\max }\right]$ and $k=[1, n]$. Then, by replacing $\alpha_{l}^{k}$ in expression $(10)$, the associated eigenvectors $\Phi_{l}(t)$ can be divided into $n$ groups $\Phi_{l}^{k}(t)$ written in the form

$$
\Phi_{l}^{k}(t)=\sum_{-j_{\max }}^{+j_{\max }} Z_{D j l}^{k} \mathrm{e}^{\mathrm{i}\left(\omega^{k}+2(l+j) \Omega\right) t}+\sum_{-j_{\max }}^{+j_{\max }} Z_{R j l}^{k} \mathrm{e}^{-\mathrm{i}\left(\bar{\omega}^{k}+2(l+j) \Omega\right) t}
$$

where $\omega^{k}$ is the fundamental frequency of $\Phi_{l}^{k}(t)$.

When $j_{\max }$ tends to infinity, the frequency spectrum width of $\Phi_{l}^{k}$ is not $l$-independent given that $Z_{j l}^{k}=A_{l} Z_{(j-1)(l+1)}^{k}$ where $A_{l}$ is any constant. Using this outstanding property in expression (12), we find that $\Phi_{l}^{k}(t)=A_{l} \Phi_{(l+1)}^{k}(t)$. In other words, the information given by the infinite set of $\Phi_{l}^{k}$ is redundant and one needs only $n$ independent eigenvectors $\Phi^{k}$ to characterize the periodically time-varying system (2) in the frequency domain. According to the linearity assumption, the Floquet solution (free whirling) is a linear combination of the quasimodes such as $z(t)=B_{k} \Phi^{k}(t)$ where $B_{k}$ is any constant.

According to Hill determinant convergence, the number of harmonic significantly contributing to the quasimodes $\Phi^{k}$, i.e. to the Floquet solution, is finite. Thus, one needs only a finite harmonic truncature order $j_{\max }$ to accurately determine their frequency spectrum. Applying Poincare's work to our rotating case, this order is determined by the series convergence

$$
\sum_{j}\left|\frac{a_{j p}}{\delta-m\left(\alpha_{l}+2 j \Omega\right)^{2}}\right| \text { with } j \neq p
$$

The convergence (13) is parametric since $j_{\max }$ depends on the rotational speed $\Omega$ and the off-diagonal coefficients $a_{j p}$, i.e. the rotating stiffness $k_{r}(t)$ through the trigonometric series (7). Hence, fast convergence is obtained for weak coupling (few $\Delta_{s}$ in (7)) or high rotational speed $\Omega$. For a given imperfection, the number of significant harmonic contributions contained in $\Phi^{k}$ decreases when $\Omega$ increases. 


\subsection{Numerical computations}

The previous concepts are illustrated by computing the parametric quasimodes of the asymmetric shaft sketched in Fig. 1. The governing equation (2) is solved in the frequency domain with the numerical data given in Section 2.3 . In the following, the given frequencies are divided by $\omega_{0}^{x}$ and also denoted by the subscript $*$.

Fig. 2 illustrates the two parametric quasimodes of Eq. (2) computed for a subcritical rotational speed $\Omega_{*}=0.34$ and a harmonic truncature order $j_{\max }=5$. Figs. 2(a) and (b) represent the orbit $\Phi^{k}(t)$ of the modal mass $m$ in the modal basis $(x, y)$ (equivalent to flexural modes in the physical one). Figs. 2(c) and (d) are the associated frequency spectrum, i.e. visualization of $\Phi^{k}$. In opposition to classic eigenmodes computed for instance at standstill, the parametric quasimodes in the $x$ and $y$ direction are poly-harmonic and complex, i.e. coupling the transverse displacements.

Fig. 3 shows the parametric quasimode and its power spectral density in the $x$ direction for a supercritical rotational speed $\Omega_{*}=4$ and a harmonic truncature order $j_{\max }=2$.

According to the convergence (13), when increasing $\Omega$, the number of significant harmonic contributions contained in the quasimodes $\Phi^{x}$ and $\Phi^{y}$ decreases. Moreover, the frequency spectra of the latter tend, respectively, to the single fundamental harmonic $\omega_{m}^{x}=\sqrt{\delta-\varepsilon / m}$ and $\omega_{m}^{y}=\sqrt{\delta+\varepsilon / m}$. In fact, the larger is the shaft rotating frequency $\Omega$ compared to its higher natural frequency $\omega_{0}^{y}$, the more the rotating stiffness seems isotropic (apparent averaging in the fixed frame). The quasimodes tend also to the equivalent classic eigenmodes of Eq. (2) with $k_{r}(t)=k_{0}$. This phenomenon highlight the quasi $\Omega$ - independence on the fundamental frequencies $\omega^{k}$ on the contrary of the strongly $\Omega$ - dependence concerning the secondary harmonics.

Remark. For a finite harmonic truncature $j_{\max }$, the relation $Z_{j l}^{k}=A_{l} Z_{(j-1)(l+1)}^{k}$ is not true for $l= \pm j_{\max }$ (truncature error). Thus, for practical purposes, the choosen parametric quasimodes $\Phi^{k}$ are the eigenvectors associated with the fundamental harmonic $\alpha_{0}^{k}=\omega^{k}$ since they converge faster than the other ones.

\subsection{Prediction of the unbalanced response}

The response to unbalance load is often required to check the capacity of a rotating machine to withstand mass and geometrical defect. Referring to Fig. 1(b), let $d$ be the distance between the mass $m$ and the rotation axis and $\phi$ the phase angle in the rotating frame. In the inertial frame, the unbalance load is a direct rotating force, proportional to the square of the constant rotational speed $\Omega^{2}$, so that the governing equation (2) becomes in the modal basis [4]

$$
m \ddot{z}(t)+c \dot{z}(t)+\delta z(t)+\varepsilon \bar{z}(t)+k_{r}(t) \bar{z}(t)=m d \Omega^{2} \mathrm{e}^{\mathrm{i}(\Omega t+\Phi)}
$$

(a)

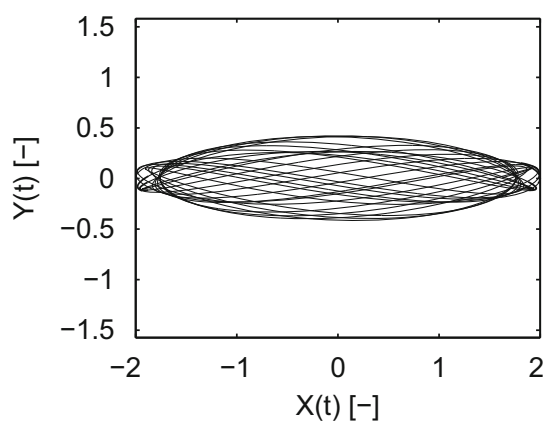

(c)

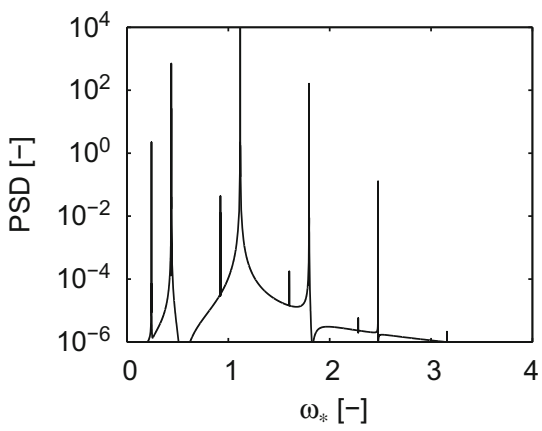

(b)

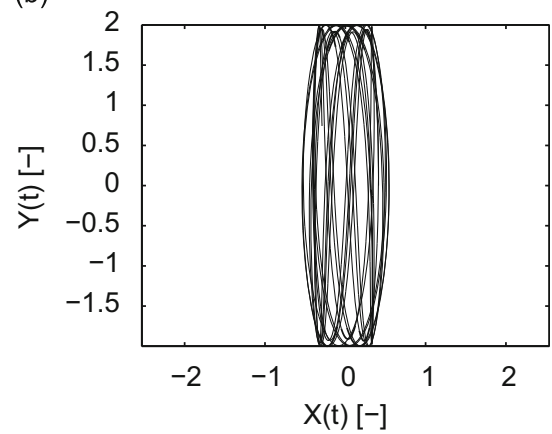

(d)

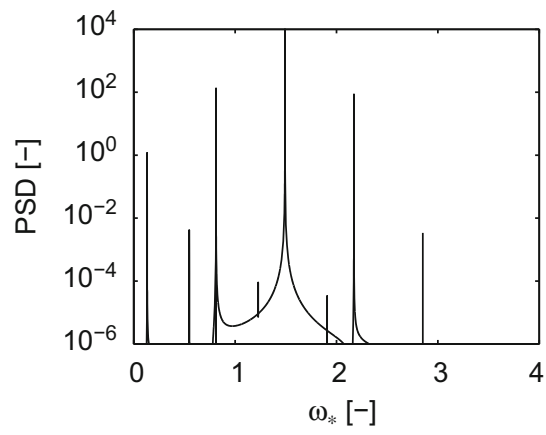

Fig. 2. Parametric quasimodes for $\Omega_{*}=0.34$ and $j_{\max }=5$ : (a) orbit of the mass for $\Phi^{x}(t)$; (b) orbit of the mass for $\Phi^{y}(t)$; (c) power spectral density of $\Phi^{x}(t)$; (d) power spectral density of $\Phi^{y}(t)$. 
(a)

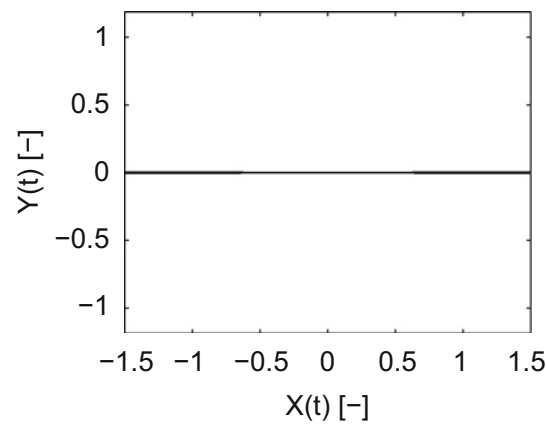

(b)

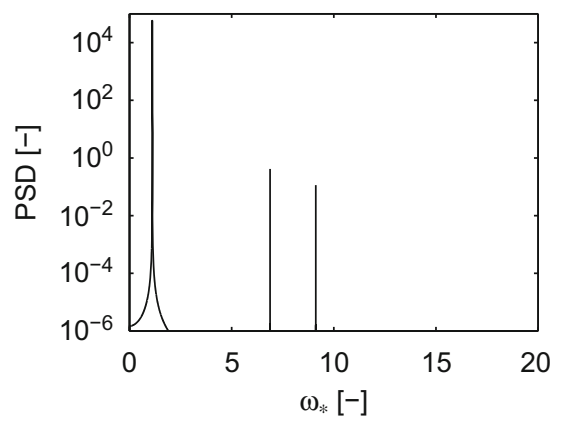

Fig. 3. Parametric quasimodes $\Phi^{x}(t)$ for $\Omega_{*}=4$ and $j_{\max }=2$ : (a) orbit of the mass; (b) power spectral density of $\Phi^{x}(t)$.

(a)

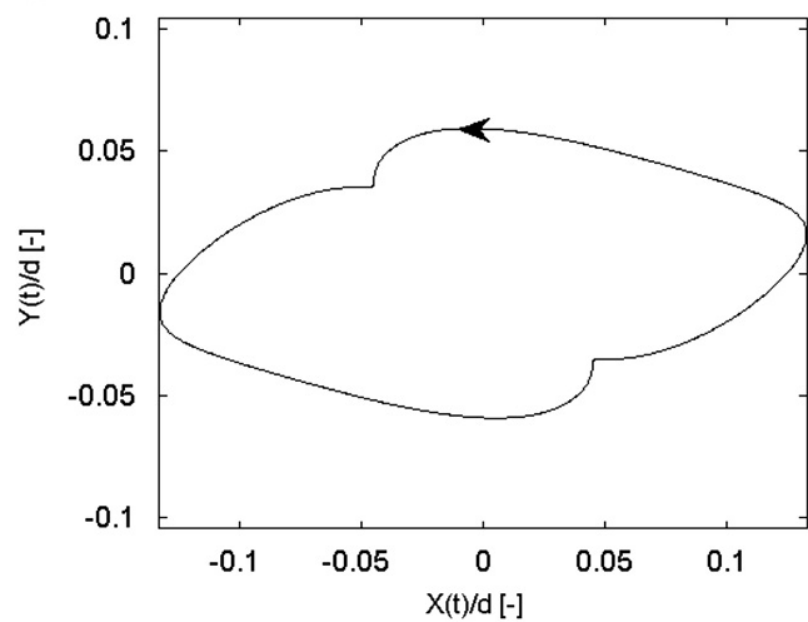

(b)

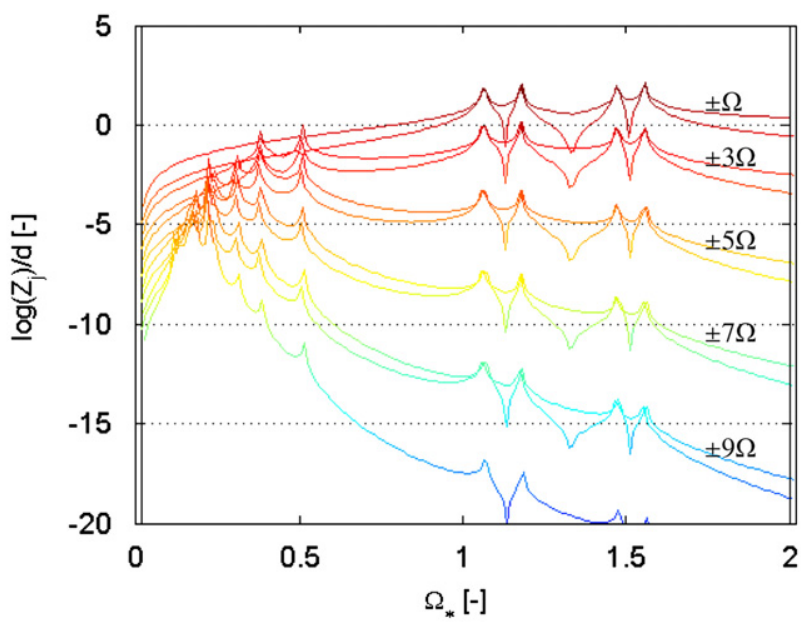

Fig. 4. Unbalance response of the horizontal asymmetric shaft in the modal basis: (a) orbit of $m$ for $\Omega_{*}=0.34$; (b) waterfall representation.

The general solution of the linear equation (14) can be written as the sum of the two particular solutions

$$
z_{p}(t)=\sum_{-j_{\max }}^{+j_{\max }} Z_{D j} \mathrm{e}^{\mathrm{i}(\Omega+2 j \Omega) t}+\sum_{-j_{\max }}^{+j_{\max }} Z_{R j} \mathrm{e}^{-\mathrm{i}(\Omega+2 j \Omega) t}
$$

By replacing, respectively, $z_{p}(t)$ and $k_{r}(t)$ by their expressions (15) and (3) in Eq. (14), unknowns $Z_{D j}$ and $Z_{R j}$ are easily computed for each $\Omega$ thanks to a truncated linear system where the matrix is equivalent to the one given in (9) with $\alpha=\Omega$. Thus, the truncature order $j_{\max }$ of expression (15) is still determined by the convergence (13) with $\alpha_{l}=\Omega$.

Fig. 4 represents the numerical computation of the unbalance response of the horizontal asymmetric shaft of Fig. 1 for $j_{\max }=5$ and $c=0.1$. The forced response is poly-harmonic in opposition to classic response of healthy shaft [3]. The waterfall in Fig. 4(b), computed for $j_{\max }=5$, shows that the steady-state response frequency spectrum is strongly dependent on the rotational speed $\Omega$ according to the convergence (13). The secondary harmonics tend to disappear when $\Omega$ is increasing. In addition to the first flexural critical speed occurring for $\Omega \approx \omega^{k}$, the vibration level of asymmetric rotating machines is characterized by secondary critical speeds due to the resonances of secondary harmonic contributions of the parametric quasimodes.

For practical purposes, according to the convergence (13), the harmonic truncature order $j_{\max }$ determined for $\Omega_{i}$ remains valid in all the domain $\left[\Omega_{i}-\Omega_{f}\right]$ if $\Omega_{i}<\Omega_{f}$.

\subsection{Particular case}

A well-known particular case occurs if the anisotropic rotor is supported by isotropic supports, i.e. when $\Delta_{r} \neq 0$ but $\varepsilon=0$. Indeed, when studying the system in the rotating frame $\mathcal{R}^{\prime}$, the governing equation is a differential equation with constant coefficients and the computation of its associated eigenproblem becomes straightforward. When working in the 
inertial frame $\mathcal{R}$, the solution of the governing equation (2) can be written in the exact form

$$
z(t)=Z_{D 0} \mathrm{e}^{\mathrm{i} \omega t}+Z_{R-1} \mathrm{e}^{-\mathrm{i}(\bar{\omega}-2 \Omega) t} .
$$

When replacing $z(t)$ in (2), the associated eigenproblem is $\Omega$ - dependent but finite and can be expressed as

$$
\left[\begin{array}{cc}
A_{-1} & -\Delta_{r} \\
-\Delta_{r} & A_{0}
\end{array}\right]\left\{\begin{array}{c}
\bar{Z}_{R-1} \\
Z_{D 0}
\end{array}\right\}=\{0\}
$$

For this particular case, Hill's method is therefore not necessary. In fact, if $\varepsilon=0$, the infinite eigenproblem (9) can be seen as $l$ independent ones in the form (17) and leads to redundant informations. Whatever $j_{\max }>0$, the relation $\Phi_{l}^{k}(t)=A_{l} \Phi_{l+1)}^{k}(t)$ is always true and Eq. (17) is sufficient to compute the $n=2$ parametric quasimodes of the rotating oscillator. According to convergence (13), the second harmonic of $z(t)$ tends to disappear when $\Omega$ increases due to the apparent rotating stiffness averaging. This case will be studied further in Section 6.

\section{Linear stability of parametric quasimodes}

\subsection{Frequency lock-in mechanism}

The Floquet solution being a linear combination of the parametric quasimodes, its stability is determined by their growth rates $\sigma_{l}$ simply related to the eigenvalues following $\sigma_{l}=-\operatorname{Im}\left(\alpha_{l}\right)$. Thus, the computation of eigenvalues of the undamped equations (9) truncated to a $j_{\max }$ order for each $\Omega$ predict the linear stability of the rotating system (Fig. 5). Referring to expressions (11) and (12), the real part of $\alpha_{l}$ gives informations on the frequencies of $z(t)$ whereas a negative imaginary part triggers its instability.

In fact, each parametric quasimode $\Phi^{k}(t)$ can be seen as the sum of forward and backward waves associated with the frequency spectra $\pm\left(\omega^{k}+2 j \Omega\right)$ and $\pm\left(\omega^{k}-2 j \Omega\right)$ where $j \in \mathbb{Z}^{+}$. According to Fig. 5(a) where only positive frequencies are represented against the rotational speed $\Omega$, there is a strong possibility of frequency lock-in between these travelling waves. The periodically time-varying rotating oscillator is then characterized by instability regions due to harmonics coalescences of parametric quasimodes.

The coalescence range depends on the harmonics concerned and the frequency lock-in phenomenon will exist only for some harmonic contributions. Concerning our asymmetric horizontal shaft, harmonics coalescences of the quasimodes are observed only for the first harmonics $\omega^{k}$ and $\omega^{k}-2 \Omega$ (Fig. 5). Instability regions including the fundamental harmonics are referred as principal instability regions while others, less prejudicial for the system, are the secondary instability regions.

\subsection{Parametric instability}

The instability of the quasimodes is parametric since it depends on the added stiffness $\Delta_{r}$ (or the rotating imperfection ratio $\alpha_{r}$ ) and the rotational speed $\Omega$. By computing the eigenproblem (9) for an order of truncature $j_{\max }=2$, we plot the most prejudicial imaginary part of the eigenfrequencies in the $\left(\alpha_{r}, \Omega_{*}\right)$ space (Fig. 6) and obtain the stability map of the system (Strutt diagram). For the undamped system in Fig. 6(a), a principal instability region is found for $\Omega \in[1-1.75]$ in

(a)

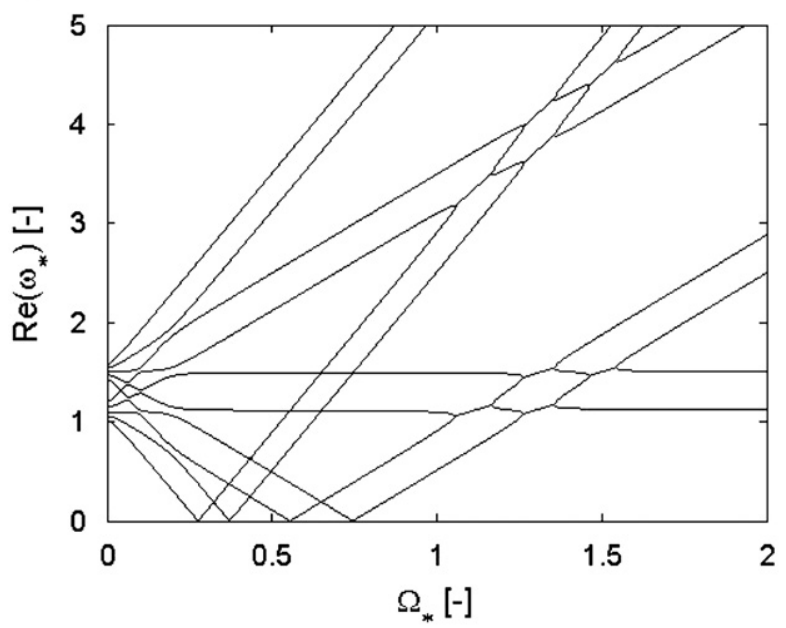

(b)

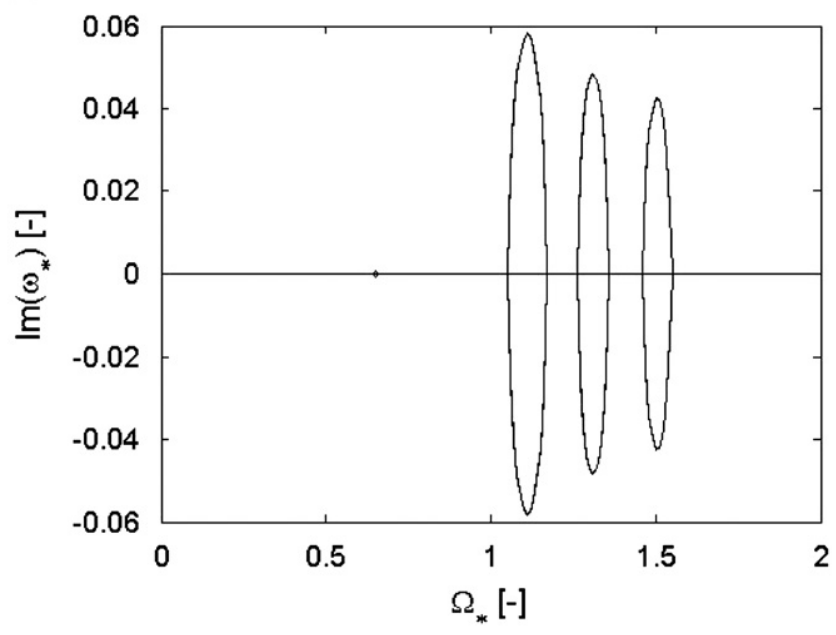

Fig. 5. Evolution of eigenvalues against rotational speed $\Omega$ for $j_{\max }=2$ : (a) real part; (b) imaginary part. 
(a)

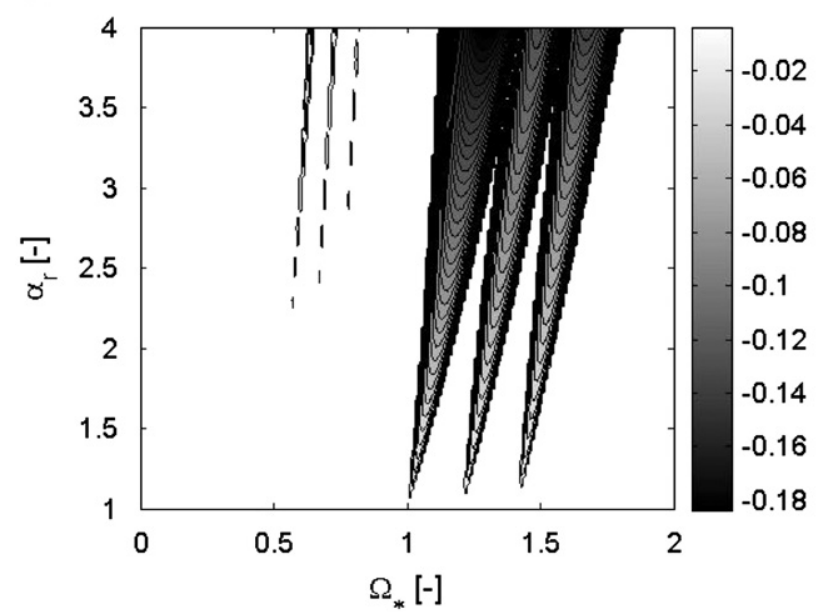

(b)

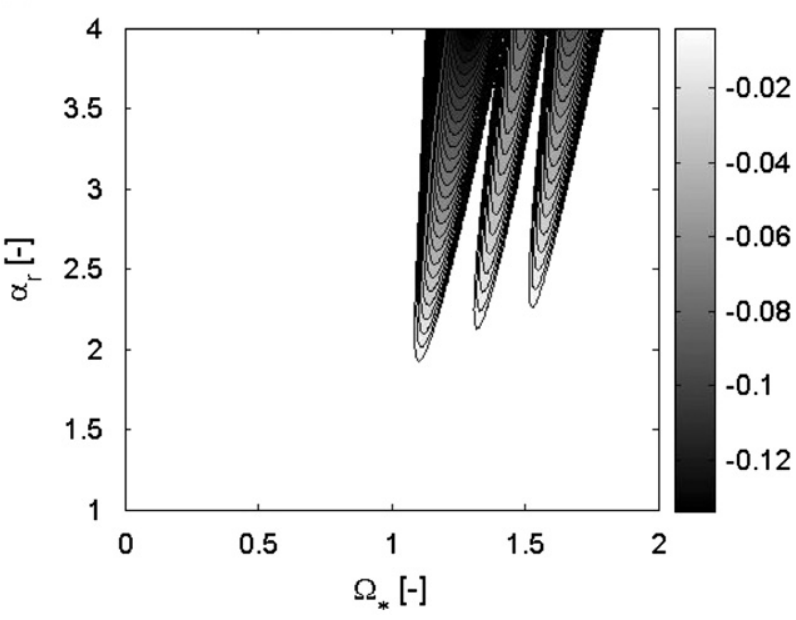

Fig. 6. Strutt diagram for $\alpha_{n}=3$ and $j_{\max }=2$ : (a) without damping $(\zeta=0)$; (b) with damping $(\zeta=0.05)$.

agreement with the Hsu criterion predicting the localization of principal instability regions for periodically time-varying linear systems with multiple degrees of freedom [18]. Moreover, a secondary instability region is visible for $\Omega \in$ [0.5-1].

The principal instability region is divided into three subdomains. The first one emerging at $\Omega=\omega_{0}^{x}\left(\Omega_{*}=1\right)$ is related to the coalescence between the fundamental and the secondary harmonic of the quasimode $\Phi^{x}(t)$. In the same way, the third one emerging at $\Omega=\omega_{0}^{y}$ results from the lock-in between the two first harmonics of the quasimode $\Phi^{y}(t)$. Finally, the intermediate subdomain is due to the cross coalescence between the first two harmonics of the different quasimodes $\Phi^{x}(t)$ and $\Phi^{y}(t)$, i.e. the fundamental harmonic of $\Phi^{x}(t)$ with the second harmonic of $\Phi^{y}(t)$ and vice versa.

For periodically time-varying linear systems with multiple degrees of freedom, parametric instabilities due to harmonic coalescences happening inside each quasimode are referred as instability of the first kind in the Hsu criterion. Frequency lock-in between two different quasimodes corresponds to the instability of the second kind and their localization depends on the symmetry of the periodic operator in the differential equation [18]. According to the Hsu criterion, the number of principal instability regions directly depends on the number of Fourier coefficients $\Delta_{s}$ in the expansion (7) of the periodic operator. Due to the weak harmonics coupling $\left(k_{r}(t)\right.$ is harmonic), there is only one principal instability region in Fig. 6 .

Finally, Fig. 6 shows that lock-in ranges are larger when increasing the rotating imperfection ratio $\alpha_{r}$. A simple solution to stabilize the system for small $\Delta_{r}$ is obtained by adding fixed damping $c$ which reduces every harmonic contributions (Fig. 6(b)). Since this effect always exists for practical purposes, we are generally not interested by the secondary instability regions.

\subsection{Particular case}

The particular case of isotropic nonrotating stiffnesses with $\varepsilon=0$ is computed through the finite eigenproblem (17) already mentioned in the previous section. Localization of the frequencies against rotational speed $\Omega$ and stability map of $z(t)$ are, respectively, given in Fig. 7 for $\zeta=0$.

The single instability region observed in Fig. 7(b) results from the coalescence between the harmonics $\omega_{0}^{x}$ and $\omega_{0}^{x}-2 \Omega$ of the quasimode $\Phi^{x}(t)$ (Fig. 7(a)). The particular Strutt diagram given in Fig. 7(b) verifies the degenerate form of the Hsu criterion when $\omega_{0}^{x}=\omega_{0}^{y}$. The lock-in range depends on $\alpha_{r}$ and is exactly located following $\Omega=\left[\omega^{x} \omega^{y}\right]$ where $\omega^{x}$ and $\omega^{y}$ are, respectively, the fundamental frequencies of the quasimodes $\Phi^{x}(t)$ and $\Phi^{y}(t)$. As in the previous general case, fixed damping would stabilize the system. Finally, note that Fig. 7(a) informs only on the localization of the harmonics of the Floquet solution. Informations about amplitudes of the harmonic contributions require the computation of the parametric quasimodes.

\section{3D finite element modelling}

\subsection{Discretization of the governing equations}

\subsubsection{Governing equations}

We consider the problem of small vibrations about the equilibrium point relative to centrifugal forces of an assembly composed of a fixed part (stator) and a rotating part (rotor). As indicated by Fig. 8, the relative speed of rotation $\Omega$ is still supposed constant in magnitude and direction. 
(a)

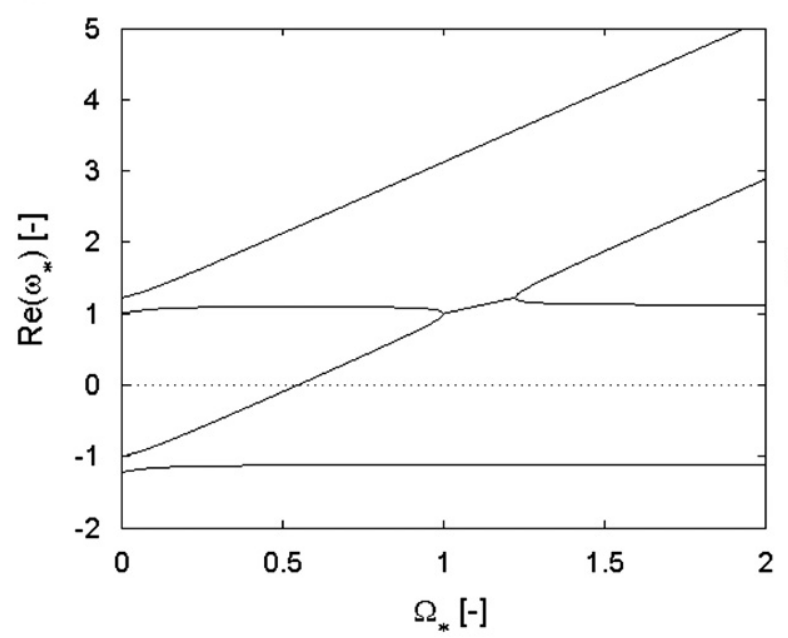

(b)

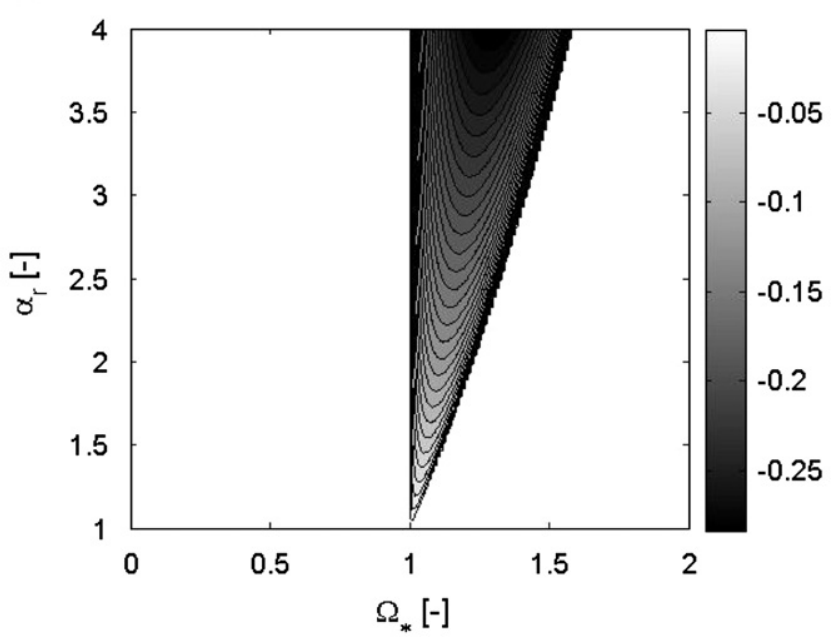

Fig. 7. Stability of the particular case $(\varepsilon=0)$ : (a) frequency spectrum against $\Omega$; (b) stability map.

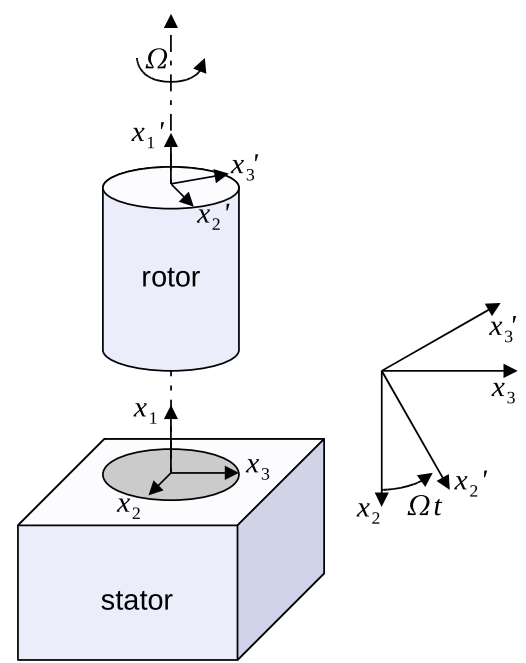

Fig. 8. Definition of the rotating (rotor) and nonrotating (stator) parts.

The absolute displacement $\mathbf{u}(\mathbf{x})$, velocity $\dot{\mathbf{u}}(\mathbf{x})$ and acceleration $\ddot{\mathbf{u}}(\mathbf{x})$ of a rotating material point $\mathbf{x}$ (with cartesian coordinates $x_{1}, x_{2}, x_{3}$ ) can be expressed in the Galilean frame $\mathcal{R}$ as function of their value in the rotating frame $\mathcal{R}^{\prime}$

$$
\begin{gathered}
\dot{\mathbf{u}}(\mathbf{x})_{\mathcal{R}}=\dot{\mathbf{u}}\left(\mathcal{R}^{\prime} / \mathcal{R}\right)+\dot{\mathbf{u}}(\mathbf{x})+\boldsymbol{\Omega} \wedge(\mathbf{x}+\mathbf{u}(\mathbf{x})) \\
\ddot{\mathbf{u}}(\mathbf{x})_{\mathcal{R}}=\ddot{\mathbf{u}}\left(\mathcal{R}^{\prime} / \mathcal{R}\right)+\ddot{\mathbf{u}}(\mathbf{x})+\dot{\boldsymbol{\Omega}} \wedge(\mathbf{x}+\mathbf{u}(\mathbf{x}))+\boldsymbol{\Omega} \wedge \boldsymbol{\Omega} \wedge(\mathbf{x}+\mathbf{u}(\mathbf{x}))+2 \boldsymbol{\Omega} \wedge \dot{\mathbf{u}}(\mathbf{x})
\end{gathered}
$$

Eqs. (18a) and (18b) contain more kinematics than those of beam elements and enable to discretize the rotor in $\mathcal{R}^{\prime}$ taking into account in particular centrifugal and Coriolis effects as discussed in [2].

We assume simple linear visco-elastic constitutive equations between the deformation (or deformation rate) and the stress tensor such as

$$
\boldsymbol{\sigma}=\boldsymbol{\sigma}^{\mathrm{el}}+\boldsymbol{\sigma}^{\mathrm{visc}}=\mathbf{D}(E, v) \boldsymbol{\varepsilon}+\mathbf{D}^{\mathrm{visc}}(\eta, v) \dot{\boldsymbol{\varepsilon}}
$$

where $\mathbf{D}$ is the Hooke matrix and $\mathbf{D}^{\text {visc }}$ is relative to the viscous part of the stress.

Then, the weak form of the problem, written for the rotating domain $\mathcal{D}$ of boundary $\left(\partial \mathcal{D}_{F} \cup \partial \mathcal{D}_{u}\right)$, reads simply

$$
\int_{\mathcal{D}} \rho \ddot{\mathbf{u}} \cdot \mathbf{v} \mathrm{d} \mathcal{D}+\int_{\mathcal{D}} \boldsymbol{\sigma}: \boldsymbol{\varepsilon}(\mathbf{v}) \mathrm{d} \mathcal{D}=\int_{\mathcal{D}} \mathbf{f}_{d} \cdot \mathbf{v} d \mathcal{D}+\int_{\partial \mathcal{D}_{F}} \mathbf{F}_{d} \cdot \mathbf{v} \mathrm{d} \Gamma
$$

$\forall \mathbf{v}$ in $V^{0}=\left\{\mathbf{v}\right.$, so that $\mathbf{v}=0$ on $\left.\partial \mathcal{D}_{u}\right\}$. For a material point $\mathbf{x}$ belonging to the stator, expression of the kinematics quantities $\mathbf{u}(\mathbf{x}), \dot{\mathbf{u}}(\mathbf{x})$ and $\ddot{\mathbf{u}}(\mathbf{x})$ in $\mathcal{R}$ are straightforward and the weak form (20) remains valid. 


\subsubsection{Discretization in the anisotropic case}

Anisotropy of both rotating and nonrotating part, interacting one with the other, leads to governing equations with periodic coefficients. In the case of open cracked rotors or for its academic counterpart rectangular rotor, a period $T=\pi / \Omega$ has to be considered. According to Floquet's theory, the form of the solution is given by

$$
\mathbf{u}(\mathbf{x}, t)=\sum_{-g_{\max }}^{g_{\max }} \mathbf{u}^{g}(\mathbf{x}) \cdot \mathrm{e}^{\mathbf{i}(\boldsymbol{\alpha}+g(2 \pi / T)) t}
$$

When applying to the rotating machine given in Fig. 8, the discretized form of rotor and stator displacements can be expressed in their respective frame as

$$
\begin{aligned}
\mathbf{u}_{\mathcal{R}^{\prime}}^{\mathrm{rot}}\left(\mathbf{X}^{\prime}, t\right) & =\sum_{-j_{\max }^{\prime}}^{+j_{\max }^{\prime}}\left[N\left(\mathbf{x}^{\prime}\right)\right]\left(\mathbf{U}^{j^{\prime}}\right)_{\mathcal{R}^{\prime}} \cdot \mathrm{e}^{\mathbf{i}\left(\omega^{\prime}+2 j^{\prime} \Omega\right) t} \\
\mathbf{u}_{\mathcal{R}}^{\mathrm{sta}}(\mathbf{x}, t) & =\sum_{-j_{\max }}^{+j_{\max }}[N(\mathbf{x})]\left(\mathbf{U}^{j}\right)_{\mathcal{R}} \cdot \mathrm{e}^{\mathbf{i}(\boldsymbol{\omega}+2 j \Omega) t}
\end{aligned}
$$

where the complex frequency notation $\boldsymbol{\omega}=\omega_{R}+\mathbf{i} \omega_{I}$ is introduced. The vectors $\mathbf{U}^{j^{\prime}}$ and $\mathbf{U}^{j}$ represent, respectively, the complex harmonic contribution $j^{\prime}$ and $j$ of $\mathbf{u}^{\text {rot }}$ and $\mathbf{u}^{\text {sta }}$ while $\omega^{\prime}$ and $\omega$ are their fundamental complex frequencies in $\mathcal{R}^{\prime}$ and $\mathcal{R}$.

Introducing in (20) the previous approximations (22) and taking into account expressions (18a), (18b) and (19), we obtain a linear system of equations in the form

$$
\sum_{-g_{\max }}^{+g_{\max }}\left[\mathbf{S}^{g}(\boldsymbol{\alpha})\right]\left(\mathbf{U}^{g}\right)=\left(\mathbf{F}^{g}\right)
$$

with

$$
\mathbf{S}^{g}=\left[\left[\mathbf{K}+\mathbf{i} 2 g \Omega \mathbf{C}-(2 g \Omega)^{2} \mathbf{M}\right]+\mathbf{i} \boldsymbol{\alpha}[\mathbf{C}+2 \mathbf{i}(2 g \Omega) \mathbf{M}]-\boldsymbol{\alpha}^{2}[\mathbf{M}]\right]
$$

and where $\mathbf{F}^{g}$ is the vector of external forces contributing to the $g$ th harmonic. Depending if the rotor $\left(g=j^{\prime}\right.$ and $\left.\boldsymbol{\alpha}=\boldsymbol{\omega}^{\prime}\right)$ or stator $(g=j$ and $\boldsymbol{\alpha}=\boldsymbol{\omega})$ is considered, the finite element matrices read

$$
\mathbf{K}=\left\{\begin{array}{l}
\mathbf{K}^{\text {rot }}+\Omega^{2} \mathbf{K}^{\mathrm{ce}}+\Omega^{2} \mathbf{K}^{\sigma} \\
\mathbf{K}^{\text {sta }}
\end{array}, \quad \mathbf{C}=\left\{\begin{array}{l}
\mathbf{C}^{\text {rot }}+\Omega \mathbf{G}^{\text {cori }} \\
\mathbf{C}^{\text {sta }}
\end{array}, \quad \mathbf{M}=\left\{\begin{array}{l}
\mathbf{M}^{\text {rot }} \\
\mathbf{M}^{\text {sta }}
\end{array}\right.\right.\right.
$$

Besides the standard stiffness, damping and mass matrices, the constant rotational speed $\Omega$ leads to specific matrices in the rotating frame $\mathcal{R}^{\prime}$ such as centrifugal stiffness $\mathbf{K}^{\text {ce }}$, geometrical stiffness $\mathbf{K}^{\sigma}$ and Coriolis pseudo-damping $\mathbf{G}^{\text {cori }}$ [2]. Finally, complete system relative to the rotor-stator assembly is obtained with kinematic relations presented in paragraph 5.2 .

\subsection{Linking rotor and stator kinematics}

Linking the parts of the rotor and the stator which interact is realized in two steps: first the displacement is condensed into spatial Fourier series, and then coefficients are equating one to another with appropriate relationships. This process considerably reduces the number of degrees of freedom involve in the interaction conditions but needs to specify, a priori, the rotor-stator global modes we are interested in.

\subsubsection{Condensation of the displacement}

As a first step, displacements of both rotating and nonrotating parts of coincident circles are condensed on a single geometrical point $\mathbf{x}$ (Fig. 9) by developing them into spatial Fourier's series.

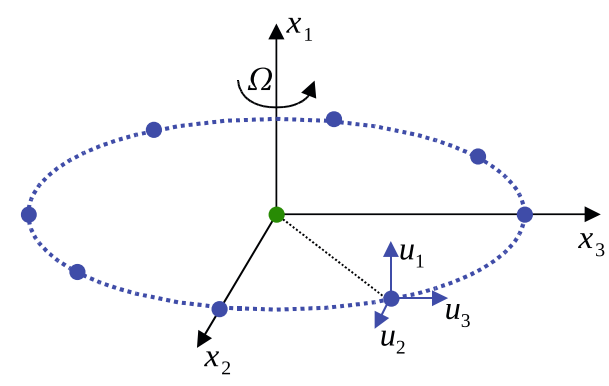

Fig. 9. Condensation of the displacement of one of the parts. 
For a considered frontier, we have

$$
\mathbf{u}(\mathbf{x}, t)=\left(\mathbf{u}(\theta) \cos \omega_{R} t-\mathbf{i u}(\theta) \sin \omega_{R} t\right) \mathrm{e}^{-\omega_{I} t} \simeq \sum_{n=0 \ldots N}\left(\left(\mathbf{U}_{n}^{c} \cos (n \theta)+\mathbf{U}_{n}^{s} \sin (n \theta)\right) \cos \omega_{R} t-\left(\mathbf{I} \mathbf{U}_{n}^{c} \cos (n \theta)+\mathbf{I} \mathbf{U}_{n}^{s} \sin (n \theta)\right) \sin \omega_{R} t\right) \mathrm{e}^{-\omega_{I} t}
$$

where the coefficients are expressed in the orthonormal basis $\left(\mathbf{e}_{1}, \mathbf{e}_{2}, \mathbf{e}_{3}\right)$ by

$$
\begin{aligned}
& \mathbf{U}_{n}^{c}=\sum_{k=1,2,3} U_{k n}^{c} \mathbf{e}_{k} \quad \text { with } U_{k n}^{c}= \begin{cases}\frac{1}{2 \pi} \int_{-\pi}^{\pi} u_{k}(\theta) \mathrm{d} \theta & \text { if } n=0 \\
\frac{1}{\pi} \int_{-\pi}^{\pi} u_{k}(\theta) \cdot \cos n \theta \mathrm{d} \theta & \text { if } n \neq 0\end{cases} \\
& \mathbf{U}_{n}^{s}=\sum_{k=1,2,3} U_{k n}^{s} \mathbf{e}_{k} \quad \text { with } U_{k n}^{s}= \begin{cases}0 & \text { if } n=0 \\
\frac{1}{\pi} \int_{-\pi}^{\pi} U_{k}(\theta) \cdot \sin n \theta \mathrm{d} \theta & \text { if } n \neq 0\end{cases}
\end{aligned}
$$

The same projection is realized for the $I U_{k n}^{c}$ part of the displacement.

Expression (24) is given in a 3D cartesian coordinate system but is obviously related to the well-known 2D-Fourier kinematics. However, because of the change of coordinate system, the coefficient $n$ is not exactly the Fourier mode number $n_{\theta}$. For instance, for imposing a condition on a rigid body motion, i.e. $n=0$, the displacement (24a) simply reduces to

$$
\mathbf{u}(\mathbf{x}, t)=\left(\mathbf{U}_{0}^{c} \cos \omega_{R} t-\mathbf{I U}_{0}^{c} \sin \omega_{R} t\right) \mathrm{e}^{-\omega_{I} t}
$$

Then, the in-plane translation in (25) is equivalent to the one of a pure flexion mode associated with $n_{\theta}=1$. On the other hand, the constant axial translation for $n=0$ correspond to an axial Fourier mode $n_{\theta}=0$.

\subsubsection{Linking the rotating and nonrotating frames}

The methodology to link the condensed displacements of rotor and stator is presented here for $n=0$, but may be applied to the general case of arbitrary $n$. Two reasons explain this choice. The first one is the sake of clarity. Indeed the introduction of a displacement depending of $\theta$ implies to make the additional change of variable $\theta^{\prime}=\theta-\Omega t$ and to use a lot of trigonometric relations to get an expression for the rotor in the same shape than the stator one. The second reason is physical. In most practical cases, rotor-bearing systems mainly act to limit rigid body motions of the shaft and other kinds of deformation (like ovalization effects for instance) are of second order.

Using the classic transition matrix and trigonometric relations, displacement of the rotor connecting point $\mathbf{x}^{\prime}$ can be expressed in the Galilean frame $\mathcal{R}$ in the expanded form

$$
\begin{aligned}
& \mathbf{u}_{\mathcal{R}}^{\mathrm{rot}}\left(\mathbf{x}^{\prime}, t\right)=\mathrm{e}^{-\omega_{I}^{\prime} t} \cdot \sum_{-j_{\max }^{\prime}}^{+j_{\max }^{\prime}}\left(\begin{array}{c}
U_{1}^{j^{\prime}} \\
0 \\
0
\end{array}\right) \cos \left(\left(\omega_{R}^{\prime}+2 j^{\prime} \Omega\right) t\right)-\left(\begin{array}{c}
I U_{1}^{j^{\prime}} \\
0 \\
0
\end{array}\right) \sin \left(\left(\omega_{R}^{\prime}+2 j^{\prime} \Omega\right) t\right)+\frac{1}{2}\left(\begin{array}{c}
0 \\
U_{2}^{j^{\prime}}-I U_{3}^{j^{\prime}} \\
U_{3}^{j^{\prime}}+I U_{2}^{j^{\prime}}
\end{array}\right) \cos \left(\left(\omega_{R}^{\prime}+\left(2 j^{\prime}+1\right) \Omega\right) t\right) \\
& -\frac{1}{2}\left(\begin{array}{c}
I U_{2}^{j^{\prime}}+U_{3}^{j^{\prime}} \\
I U_{3}^{j^{\prime}}-U_{2}^{j^{\prime}}
\end{array}\right) \sin \left(\left(\omega_{R}^{\prime}+\left(2 j^{\prime}+1\right) \Omega\right) t\right)+\frac{1}{2}\left(\begin{array}{c}
0 \\
U_{2}^{j^{\prime}}+I U_{3}^{j^{\prime}} \\
U_{3}^{j^{\prime}}-I U_{2}^{j^{\prime}}
\end{array}\right) \cos \left(\left(\omega_{R}^{\prime}+\left(2 j^{\prime}-1\right) \Omega\right) t\right)-\frac{1}{2}\left(\begin{array}{c}
0 \\
I U_{2}^{j^{\prime}}-U_{3}^{j^{\prime}} \\
I U_{3}^{j^{\prime}}+U_{2}^{j^{\prime}}
\end{array}\right) \sin \left(\left(\omega_{R}^{\prime}+\left(2 j^{\prime}-1\right) \Omega\right) t\right)
\end{aligned}
$$

which can be recast in a more compact way

$$
\mathbf{u}_{\mathcal{R}}^{\text {rot }}\left(\mathbf{x}^{\prime}, t\right)=\sum_{-j_{\max }^{\prime}}^{+j_{\max }^{\prime}} \mathbf{U}_{A}^{j^{\prime}} \mathrm{e}^{\left.\left(\omega^{\prime}+2 j^{\prime} \Omega\right) t\right)}+\mathbf{U}_{F}^{j^{\prime}} \mathrm{e}^{\left(\omega^{\prime}+\left(2 j^{\prime}+1\right) \Omega\right) t}+\mathbf{U}_{B}^{j^{\prime}} \mathrm{e}^{\left(\omega^{\prime}+\left(2 j^{\prime}-1\right) \Omega\right) t}
$$

and where subscript $A, F$ and $B$, respectively, denotes axial, forward and backward motions. The relationship between the natural frequencies in the rotating and fixed frames is function of the investigated Fourier mode number following $\omega^{\prime}=\omega-n_{\theta} \Omega$ [2]. For $n=0$ and according to the previous remark, the relation becomes $\omega^{\prime}=\omega$ for an axial motion and $\omega^{\prime}=\omega-\Omega$ for an in-plane displacement. Thus, complex oscillations of the rotor connecting point can be expressed in the stator coordinate system such as

$$
\mathbf{u}_{\mathcal{R}}^{\mathrm{rot}}(\mathbf{x}, t)=\sum_{-j_{\max }^{\prime}}^{+j_{\max }^{\prime}} \mathbf{U}_{A}^{j^{\prime}} \mathrm{e}^{\left.\left(\omega+2 j^{\prime} \Omega\right) t\right)}+\mathbf{U}_{F}^{j^{\prime}} \mathrm{e}^{\left(\omega+2 j^{\prime} \Omega\right) t}+\mathbf{U}_{B}^{j^{\prime}} \mathrm{e}^{\left(\omega+2\left(j^{\prime}-1\right) \Omega\right) t}
$$

On the other side, the expanded condensed displacement of the stator part is recast for convenience in the form

$$
\mathbf{u}_{\mathcal{R}}^{\mathrm{sta}}(\mathbf{x}, t)=\sum_{j_{\max }}^{+j_{\max }} \mathbf{U}_{A}^{j} \mathrm{e}^{(\omega+2 j \Omega) t)}+\mathbf{U}_{F}^{j} \mathrm{e}^{(\omega+2 j \Omega) t}+\mathbf{U}_{B}^{j} \mathrm{e}^{(\omega+2 j \Omega) t}
$$


By simply equating the two expressions (27) and (28) for each harmonic, it appears the kinematic relations modelling the harmonic coupling due to rotor-stator interaction limited to the displacements verifying $n=0$

$$
\mathbf{U}_{A}^{j}=\mathbf{U}_{A}^{j^{\prime}}=j, \quad \mathbf{U}_{F}^{j}=\mathbf{U}_{F}^{j^{\prime}}=j, \quad \text { and } \quad \mathbf{U}_{B}^{j}=\mathbf{U}_{B}^{\mathbf{j}^{\prime}}=j+1
$$

These relations have to be fulfil for the truncature order $j \in\left\{-j_{\max } \ldots j_{\max }\right\}$ and $j^{\prime} \in\left\{-j_{\max }^{\prime} \ldots j_{\max }^{\prime}\right\}$. Moreover the boundary conditions $\mathbf{U}_{B}^{j=j_{\max }}=0$ and $\mathbf{U}_{B}^{j^{\prime}}=-j_{\max }=0$ should not be forgotten.

To capture an higher kinematics such as $n>0$ (disc modes, ovalization modes, etc.), the process described here remains relevant, the difference being the stronger difficulty to express the equations. The supplementary kinematic relations associated with a $n$ Fourier coefficients are simply added to the previous boundary conditions.

\subsection{Taking advantage of substructuring and modal synthesis}

Eqs. (22a) and (22b) indicate that the same discretization is used for every terms of the Hill expansion. The set of vectors $\left\{\mathbf{U}^{\prime}, \mathbf{U}^{\prime}\right\}$ are linked one to another only by the kinematic relations exposed in paragraph 5.2. These basic observations allow us to take great advantage of substructuring and modal synthesis methods. Craig-Bampton substructuring [19] is therefore adapted to the case of study.

\subsubsection{Substructuring}

For every harmonic $j^{\prime}$ and $j$, the corresponding vector of unknown harmonic contributions is approximated by the Ritz transformation

$$
\begin{gathered}
\mathbf{U}^{j^{\prime}} \simeq\left[\begin{array}{ll}
\boldsymbol{\Phi}_{\alpha}^{j^{\prime}} & \boldsymbol{\Phi}_{\beta}^{j^{\prime}}
\end{array}\right] \cdot\left(\begin{array}{c}
\mathbf{q}_{\alpha}^{j^{\prime}} \\
\mathbf{q}_{\beta}^{j^{\prime}}
\end{array}\right) \simeq\left[\boldsymbol{\Phi}^{j^{\prime}}\right] \cdot\left(\mathbf{q}^{j^{\prime}}\right) \\
\mathbf{U}^{j} \simeq\left[\begin{array}{ll}
\boldsymbol{\Phi}_{\alpha}^{j} & \boldsymbol{\Phi}_{\beta}^{j}
\end{array}\right] \cdot\left(\begin{array}{c}
\mathbf{q}_{\alpha}^{j} \\
\mathbf{q}_{\beta}^{j}
\end{array}\right) \simeq\left[\boldsymbol{\Phi}^{j}\right] \cdot\left(\mathbf{q}^{j}\right)
\end{gathered}
$$

where $\boldsymbol{\Phi}_{\alpha}^{j^{\prime}}=\left[\boldsymbol{\phi}_{\alpha 1}^{\prime} \ldots\right]$ (resp. $\boldsymbol{\Phi}_{\alpha}^{j}=\left[\boldsymbol{\phi}_{\alpha 1} \ldots\right]$ ) refers to the set of fixed interface normal modes obtained for the undamped rotating (resp. nonrotating) part of the system, and $\boldsymbol{\Phi}_{\beta}^{j^{\prime}}=\left[\boldsymbol{\phi}_{\beta 1}^{\prime} \ldots\right]$ (resp. $\boldsymbol{\Phi}_{\beta}^{j}=\left[\boldsymbol{\phi}_{\beta 1}^{\prime} \ldots\right]$ ) refers to the set of constraint modes obtained as static solutions of the rotating (resp. nonrotating) part of the system.

The complex modes $\boldsymbol{\Phi}^{j^{\prime}}$ and $\boldsymbol{\Phi}^{j}$, identical for every $j^{\prime}$ and $j$, are calculated only one time according to

$$
\begin{gathered}
{\left[\mathbf{K}-\omega_{m}^{2} \mathbf{M}+\mathbf{B}\right] \cdot\left(\boldsymbol{\phi}_{\alpha m}\right)=(0)} \\
\left.\left[\begin{array}{cc}
\mathbf{K} & \mathbf{B}_{n}^{T} \\
\mathbf{B}_{n} & 0
\end{array}\right] \cdot\left(\begin{array}{c}
\boldsymbol{\phi}_{\beta n} \\
\lambda_{n}
\end{array}\right)=\begin{array}{c}
0 \\
\mathbf{U}_{n}^{\mathrm{imp}}=1
\end{array}\right)
\end{gathered}
$$

where $\mathbf{K}$ and $\mathbf{M}$ are the classic stiffness and mass matrix of the rotor or stator (for $\Omega=0$ ). $\mathbf{B}_{n}$ refers to the boundary conditions matrix of the $n$th interface degree of freedom and $\mathbf{B}$ gathers every $\mathbf{B}_{n}$. The static modes $\boldsymbol{\phi}_{\beta n}$ are obtained by imposing successively a unit displacement on each generalized coordinates $\mathbf{q}_{\beta}$ which are simply the coefficients $\mathbf{U}_{n}$ of the spatial Fourier series (24) used in the previous condensation.

\subsubsection{Modal synthesis}

The modal basis of the global system incorporating all the harmonic contributions of both rotor and stator (and the kinematic relations linking one to another) is computed with the modal synthesis method at standstill, and for an order of truncature $j_{\max }$, according to

$$
\left(\overline{\mathbf{K}}-\omega^{2} \overline{\mathbf{M}}\right) \mathbf{q}+\mathbf{L}^{\mathbf{j}_{\max }^{T}} \boldsymbol{\lambda}=(\mathbf{0}) \text { and } \quad \mathbf{L}^{j_{\max }} \mathbf{q}_{\beta}=(\mathbf{0})
$$

The entities $\overline{\mathbf{K}}$ and $\overline{\mathbf{M}}$ are the stiffness and mass matrix of the complete system built with the modes $\boldsymbol{\Phi}^{j^{\prime}}$ and $\boldsymbol{\Phi}^{j}$ for $j \in\left\{-j_{\max } \ldots j_{\max }\right\}$ and $j^{\prime} \in\left\{-j_{\max }^{\prime} \ldots j_{\max }^{\prime}\right\}$. The generalized coordinates $\mathbf{q}$ (or the interface coordinates $\mathbf{q}_{\beta}$ ) are in the form

$$
\mathbf{q}=\left[\begin{array}{lllll}
\mathbf{q}^{-j_{\max }^{\prime}} & \mathbf{q}^{-j_{\max }} & \ldots & \mathbf{q}^{j_{\max }^{\prime}} & \mathbf{q}^{j_{\max }}
\end{array}\right]^{T}
$$

The matrix of boundary conditions $\mathbf{L}^{j_{\max }}$ arises from the previous Section 5.2. Notably, if only interactions $n=0$ are considered, $\mathbf{L}^{j_{\max }}$ is expressed, according to (29), in the form

$$
\mathbf{L}^{j_{\max }}=\left[\begin{array}{cccccc}
- & - & - & - & - & - \\
- & \mathbf{L}_{B}^{-1} & \mathbf{L}_{B}^{0^{\prime}} & 0 & 0 & - \\
- & 0 & \mathbf{L}_{A+F}^{0^{\prime}} & \mathbf{L}_{A+F}^{0} & 0 & - \\
- & 0 & 0 & \mathbf{L}_{B}^{0} & \mathbf{L}_{B}^{1^{\prime}} & - \\
- & - & - & - & - & -
\end{array}\right]
$$


The eigenproblem (32) provides the modal basis $\boldsymbol{\Phi}_{s s}$ allowing the global vibration analysis of a complete 3D rotating machine. The parametric quasimodes are obtained by solving the homogeneous part of (23)

$$
\sum_{-g_{\max }}^{+g_{\max }}\left[\mathbf{S}^{g}(\omega, \Omega)\right]\left(\mathbf{U}^{g}\right)=(0)
$$

projected on $\boldsymbol{\Phi}_{s s}$. This parametric eigenproblem simulates the harmonic coupling of the Floquet solution through the modal basis $\boldsymbol{\Phi}_{s s}$. It is directly expressed in the working frame $\mathcal{R}$ stating $\omega^{\prime}=\omega-n_{\theta} \Omega$ and needs also to know, a priori, the kinematics of the investigated modes.

On the one hand, the 3D quasimodes orbits are reconstructed in the Galilean frame $\mathcal{R}$ for each $\Omega$ according to the Hill expansion (22). On the other hand, their linear parametric stability are calculated by analysing the imaginary part of the eigenvalues $\operatorname{Im}(\omega)$ in a specific rotational speed range. Moreover, vibration levels of the machine under external loads (unbalancing, weight, etc.) are predicted thanks to the complete steady-state problem (22) expressed in $\mathcal{R}$ and still projected on the modal basis $\boldsymbol{\Phi}_{s s}$.

Applying Eq. (33) to the two degrees of freedom problem studied in the previous section shows that (33) is only a reformulation of the eigenproblem (9) in a more convenient way for the 3D modelling. As a consequence, the harmonic convergence is still governed by Poincare's formula (13) and $g_{\max }$ depends on the rotating speed $\Omega$ and the stiffness anisotropy introduced in $\overline{\mathbf{K}}$.

\section{Application to an academic test bench}

\subsection{Experimental setup}

In this section, an academic test bench is studied to demonstrate the capacity of the proposed method to predict the dynamical behaviour of an asymmetric rotating machine. The experimental mockup consists in a vertical rectangular cross-section shaft mounted on an anisotropic stator. Fig. 10(a) shows a 3D modelling of the test bench. The steel structure is designed, so that flexible rotor and stator interact enough to observe important secondary harmonics in the vibratory response.

The fixed part is composed of a quasirigid plate supported by four rectangular flexible struts (length $0.21 \mathrm{~m}$ and section $0.0119 \times 0.007 \mathrm{~m}^{2}$ ). The rectangular shaft (length $0.60 \mathrm{~m}$ and section $0.034 \times 0.024 \mathrm{~m}^{2}$ ) is mounted using a pair of conical bearings and is driven by an asynchronous motor working in the frequency range $\Omega=[0-50] \mathrm{Hz}$. The mass of the stator is $23 \mathrm{~kg}$ while the rotor one is $7.2 \mathrm{~kg}$.

(a)

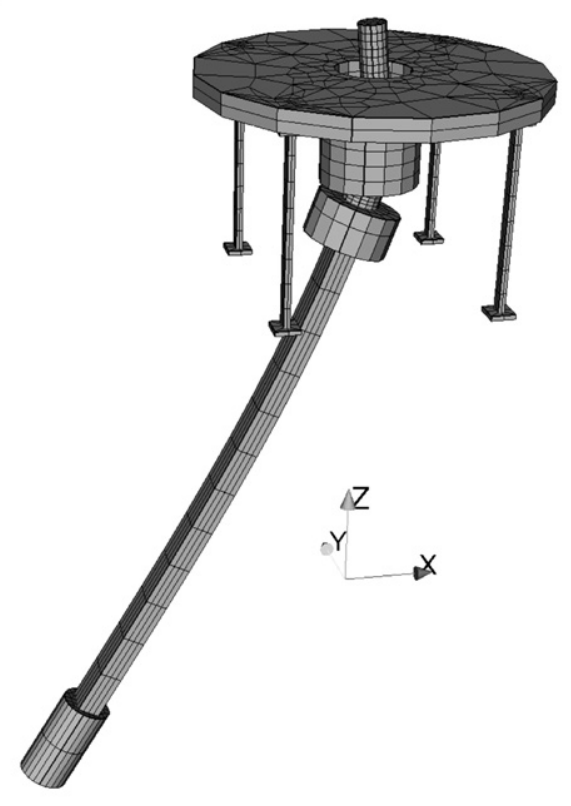

(b)

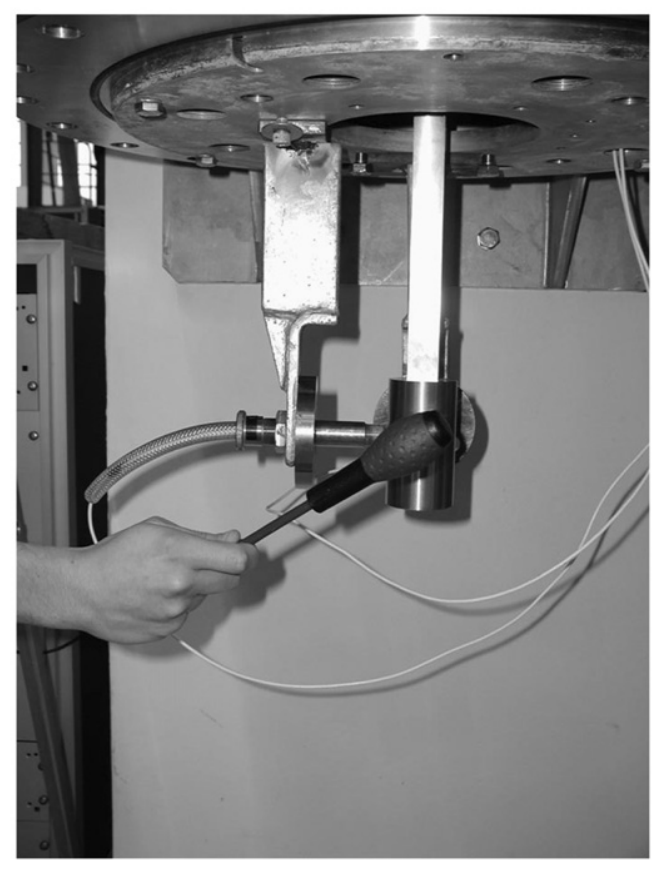

Fig. 10. Description of the academic test bench: (a) first flexural mode in the particular case; (b) vibration excitator and sensors. 
Whirling motion of the rotor is sensed using two orthogonal non-contacting eddy-current displacement sensors fixed in $\mathcal{R}$ along the $x$ and $y$ directions and, respectively, called $d B x$ and $d B y$. Both are placed around the cylindrical mass located at the end of the shaft (Fig. 10). Free whirling features are obtained by applying a random brief impact on the cylindrical mass in a direction about $45^{\circ}$ with the $x$ and $y$ axes (Fig. 10(b)). For each investigated spin speed $\Omega$, the linear response signal is captured and recorded by the sensors. Experimental results consist of the average of 10 recordings. The free whirling frequency spectrum is finally obtained by using a simple signal processing system and modal analysis software.

Two experimental setup are investigated in the following subsections. In the first particular case, the displacements of the supporting plate are forbidden. In the second case, the supporting plate is free to move. For both, comparison of the experimental results with the numerical ones is provided. Numerical results are obtained with the Finite Element freeware Cast3m [20].

\subsection{Particular case: nonisotropic rotor on isotropic support}

When the support is restrained to immobility, the test bench behaves as a simple rectangular shaft mounting on isotropic fixed bearings.

At standstill $(\Omega=0 \mathrm{~Hz})$, the two first flexural beam modes of the rotor associated with the natural frequencies $\omega_{1}=20 \mathrm{~Hz}$ and $\omega_{2}=22.5 \mathrm{~Hz}$ are identified (dashed lines in Fig. 11(a)). Flexion mode along the $x$ axis is represented in Fig. 10(a). The kinematics of these two first modes is sufficient to capture the global dynamic behaviour of the rotating oscillator in the motor working range $[0-50] \mathrm{Hz}$.

For $\Omega>0$, Fig. 11(a) clearly shows the emergence of two secondary frequencies in the free whirling response. By reporting the experimental frequency spectra of the free whirling motion for a set of $\Omega$, the evolution of the frequencies location against $\Omega$ can be plotted in Fig. 11(b). When the fundamental frequencies slowly tend to a unique average frequency $\omega=\left(\omega_{2}-\omega_{1}\right) / 2$ with $\Omega$, the secondary harmonics strongly depend on it following $\omega=\omega_{1}-2 \Omega$ and $\omega=\omega_{2}+2 \Omega$. Moreover, when increasing $\Omega$, the secondary harmonics magnitudes decrease while the fundamental ones remain almost constant. Experimental results are limited to the rotational speed $\Omega=1020 \mathrm{rev} / \mathrm{min}(17 \mathrm{~Hz})$ because of the risky vibratory levels observed from this speed.

Understanding the dynamical behaviour of the rectangular shaft in rotation is achieved with the help of the 3D finite element method presented in the previous section. In this particular case, only the rotor is considered in the rotating frame $\mathcal{R}^{\prime}$ and the conical bearings are modelled by simply clamping the shaft. At standstill, the computation of the classic eigenmodes is easy and leads to the two first flexural modes of natural frequencies $\omega_{1}=20 \mathrm{~Hz}$ and $\omega_{2}=22.5 \mathrm{~Hz}$. The higher modes (second flexural modes, twisting modes, etc.) are neglected because too distant from the range of interest [0-50] Hz.

To take into account the shaft asymmetry when rotating, the first step is to compute the extended modal basis $\boldsymbol{\Phi}_{s s}$. As introduced in the previous section, $\boldsymbol{\Phi}_{s s}$ results from the eigenproblem (32) expressed at standstill and solved with the modal synthesis method. In the particular case considered here, the only substructures modelling the harmonic contributions comes from the rotor. Linking rotating and nonrotating frame is achieved by applying the boundary conditions matrix $\mathbf{L}^{j_{\max }}$ between the harmonic contributions of the condensed displacement in $\mathcal{R}^{\prime}$ and a virtual node modelling the Galilean frame $\mathcal{R}$. The Fourier number $n=0$ being sufficient for the global modes investigated, the matrix $\mathbf{L}^{j_{\max }}$ is the same as the one expressed in (32). According to Sections 3.5 and 4.3, the eigenproblem dimension is finite in the

(a)

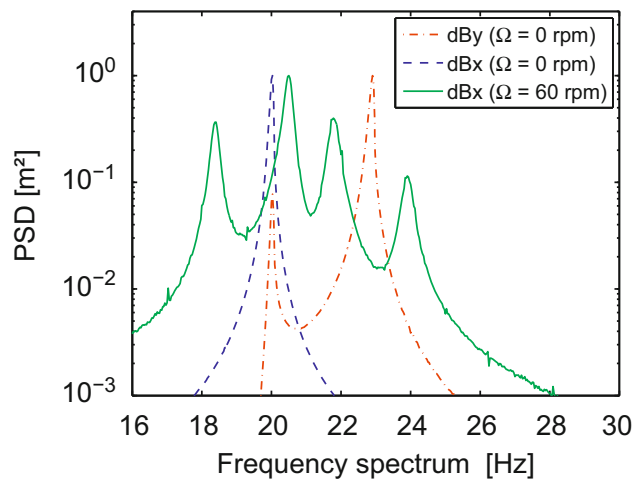

(b)

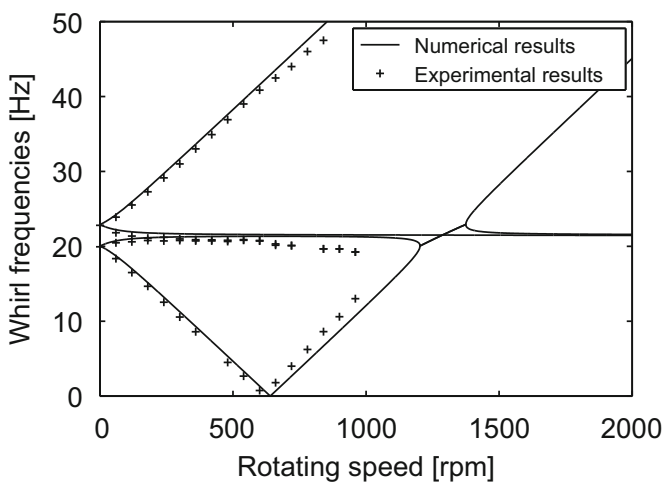

Fig. 11. Free whirling frequency response in the particular case: (a) experimental power spectral density against frequency; (b) evolution of natural frequencies location against $\Omega$. 
frequency domain. Thus, only the harmonics $j=0,-1$ and $j^{\prime}=0$ are needed to compute $\boldsymbol{\Phi}_{s s}$ and $\mathbf{L}^{j_{\max }}$ becomes

$$
\mathbf{L}^{j_{\max }}=\mathbf{L}^{p}=\left[\begin{array}{ccc}
\mathbf{L}_{B}^{-1} & \mathbf{L}_{B}^{0^{\prime}} & 0 \\
0 & \mathbf{L}_{A+F}^{0^{\prime}} & \mathbf{L}_{A+F}^{0} \\
0 & 0 & \mathbf{L}_{B}^{0}
\end{array}\right]
$$

The quasimodes of the rotating shaft are computed for the desired $\Omega$ by projecting the eigenproblem (23) on the previous modal basis $\boldsymbol{\Phi}_{s s}$. The 3D quasimodes motion is then reconstructed in the temporal domain thanks to Hill expansion (21). These computations lead to two quasimodes with no prevailing axis: a forward mode waving in the rotation direction and a backward one waving in the opposite direction. Figs. 12(a) and (b) represent, respectively, the orbit in $\mathcal{R}$ of a circumferential point of the rotating cylindrical mass for a forward and backward quasimode computed at $\Omega=60 \mathrm{rev} / \mathrm{min}(1 \mathrm{~Hz}$ ). Their power spectral density (PSD) plotted in Fig. 12(c) shows two fundamental frequencies around $\omega_{1}$ and $\omega_{2}$, and two secondary ones located at $\omega=\omega_{1}-2 \Omega$ and $\omega=\omega_{2}+2 \Omega$. Note that rotational frequency is present as well as in the figure. Free whirling being a linear combination of the quasimodes, these later give informations on the magnitude ratio between harmonic contributions which is in good agreement with experimental ratio observed in Fig. 11(a).

By computing the projected eigenproblem (23) for several $\Omega$, we plot the evolution of the shaft frequencies in Fig. 11(b). Numerical predictions are found very close to the experimental values. According to Poincare's convergence, the secondary harmonic contributions decrease when $\Omega$ increases. The quasimodes tend then to the classic modes of an isotropic circular shaft of frequency $\omega=\left(\omega_{2}-\omega_{1}\right) / 2$ which is in agreement with the experimental results. Finally, the high vibratory

(a)

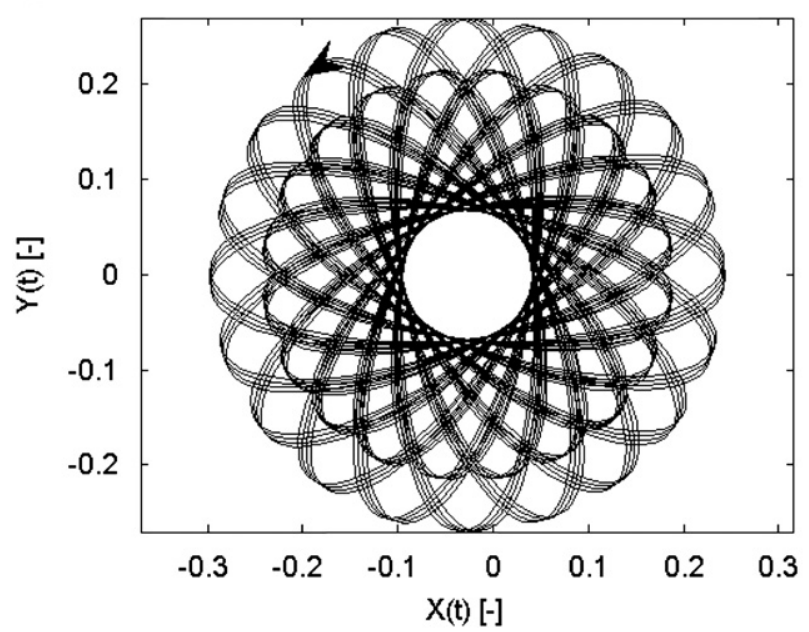

(b)

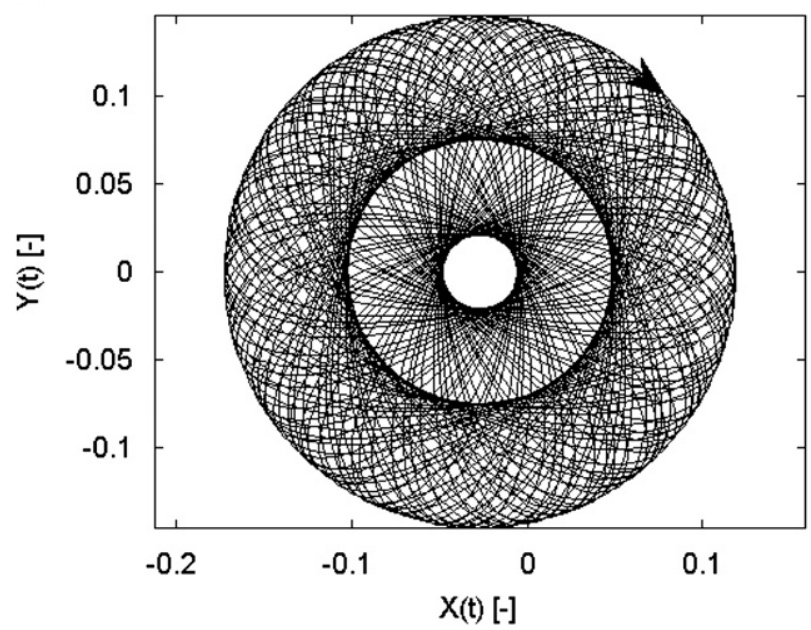

(c)

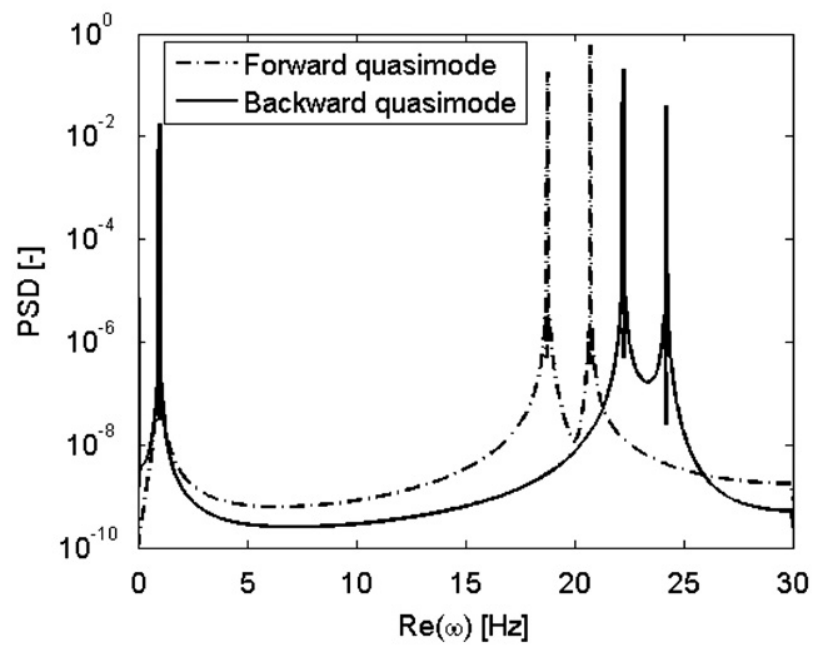

Fig. 12. Parametric quasimodes computed for $\Omega=60 \mathrm{rev} / \mathrm{min}$ in the particular case: (a) orbit of the forward quasimode; (b) orbit of the backward quasimode; (c) associated frequency spectra. 
amplitude limiting the experiments observed for $\Omega \approx 1020 \mathrm{rev} / \mathrm{min}$, can be explained by the numerical model: it is most probably due to the imminence of a lock-in phenomena between the two harmonics of the forward quasimode (Section 4.3).

\subsection{General case: anisotropy of rotor and support}

When the supporting plate is free to move, the flexible fixed rectangular struts implicitly interact with the flexible rectangular shaft and the whole rotating machine behaves as a periodic time-varying system whatever the choosen working frame.

At standstill, experimental setup brings out four global modes with natural frequencies included in the motor working range [0-50] Hz. Two supplementary orthogonal sensors located at the plate level allow to discern the global flexural mode where the plate and the cylindrical mass oscillate in phase from the modes where they are out of phase. Due to the system anisotropy, the in-phase (ip) modes and out-of-phase (op) ones have different natural frequencies along the $x$ and $y$ directions. Fig. 14(a) is a model of the in-phase and out-of-phase mode along the $x$ axis. When the rotor angular position is set, so that the second moment of area of the whole system is minimal along the $x$ axis, the natural frequencies read $\omega_{x}^{i p}=15 \mathrm{~Hz}, \omega_{y}^{i p}=21 \mathrm{~Hz}, \omega_{x}^{o p}=23 \mathrm{~Hz}$ and $\omega_{y}^{o p}=31 \mathrm{~Hz}$.

The frequency spectrum processed from the signal response captured by the shaft non-contacting sensor along the $x$ direction is given in Fig. 14 for the rotational speeds $\Omega=7$ and $8 \mathrm{~Hz}$. These experimental results are filtered to remove the spin speed subharmonics and bearing frequencies. As expected from the rotor-stator interactions, supplementary harmonics emerge in the free whirling response. The subharmonics are simply located since they follow the relation $\omega_{j} \approx \omega_{f} \pm 2 j \Omega$ where $\omega_{f}$ are the fundamental frequencies being almost the natural frequencies at standstill. In Fig. 13, fundamental and first secondary harmonics related to the natural frequencies $\omega_{x}^{i p}$ and $\omega_{x}^{o p}$ are, respectively, marked with red square marks and with blue spots. For the considered spin speed, harmonics associated with $j>1$ are not sensed anymore. Finally, note that fundamental frequencies $\omega_{y}^{i p}$ and $\omega_{y}^{o p}$ are not sensed by $d B x$ while their associated secondary harmonics are present in the frequency spectra.

Because of the number of harmonics and according to the relation $\omega_{j}=\omega_{f} \pm 2 j \Omega$, it is hazardous to made out the free whirling frequency spectrum at small rotational speed. Evolution of experimental frequencies are therefore not plotted for the general case. However, the rotating stiffness averaging for high $\Omega$ and the related magnitude decreasing of the secondary harmonics are still experimentally observed for the exploitable spin speed range.

Once again, the global dynamic behaviour of the experimental test bench is modelled with the 3D finite element method previously described. The flexible stator (plate + struts) is described in $\mathcal{R}$ while the rotor is still modelled in $\mathcal{R}^{\prime}$. Contrary to the previous case, the shaft is no more clamped in since the conical bearings transmit the rigid body motions between the rotor and stator. This interaction is taken into account by linking condensed displacements of rotor and stator at the bearings level according to the Fourier expansion for $n=0$ given in (24).

At standstill, the classic eigenmodes are obtained by simply equating the real part of the condensed displacements between the rotor and stator connecting part. In the working range [0-50] Hz, the computed modes are the four flexural global modes highlighted in the experiments with the frequencies $\omega_{x}^{i p}, \omega_{y}^{i p}, \omega_{x}^{o p}$ and $\omega^{o p}$. The in-phase and out-of-phase shapes along the $x$ axis are given in Fig. 14 .

The extended modal basis $\boldsymbol{\Phi}_{s s}$ is computed in the frequency domain and $\mathcal{R}$ following (32). In this case, the stiffness and mass matrix of the modal synthesis method are build with the complete $j_{\max }$ stator and $j_{\max }^{\prime}$ rotor substructures. The

(a)

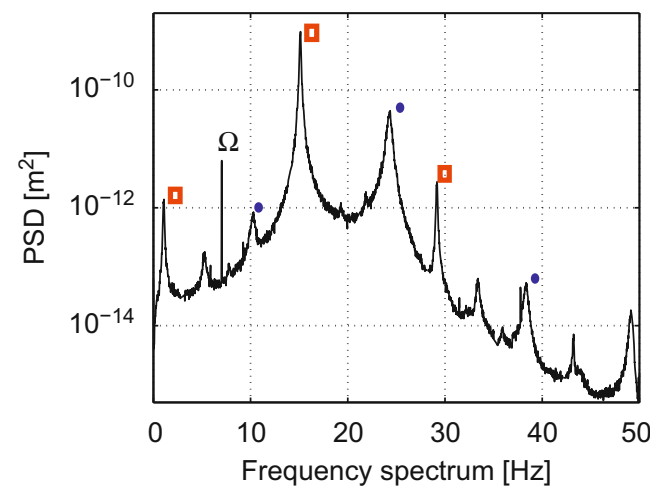

(b)

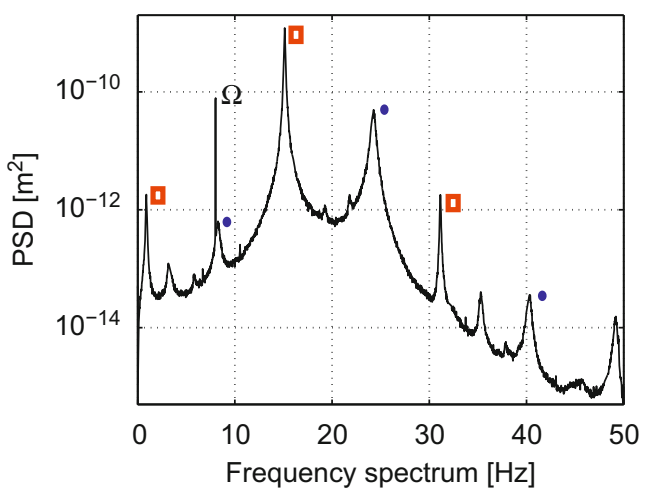

Fig. 13. Experimental $d B x$ signal frequency spectra in the general case: (a) for $\Omega=420 \mathrm{rev} / \mathrm{min}$ ( $7 \mathrm{~Hz}$ ); (b) for $\Omega=480 \mathrm{rev} / \mathrm{min}$ ( $8 \mathrm{~Hz}$ ). Harmonics related to the natural frequencies $\omega_{x}^{i p}$ and $\omega_{x}^{o p}$ are, respectively, marked with red square marks and with blue spots. (For interpretation of the references to colour in this figure legend, the reader is referred to the web version of this article.) 
(a)

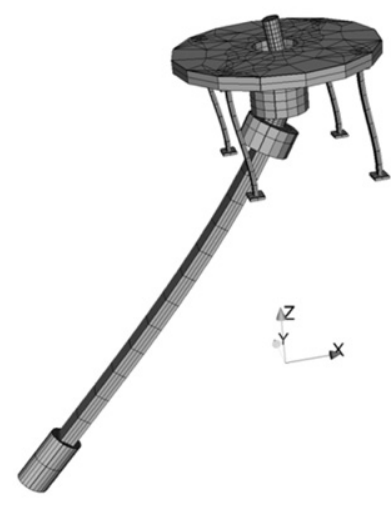

(b)

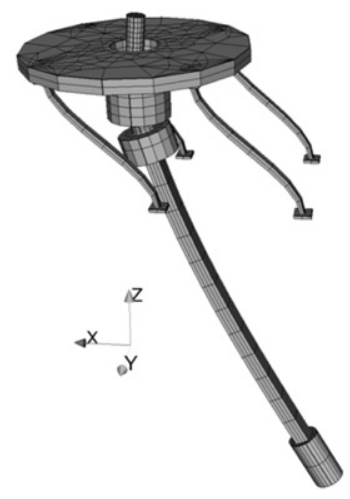

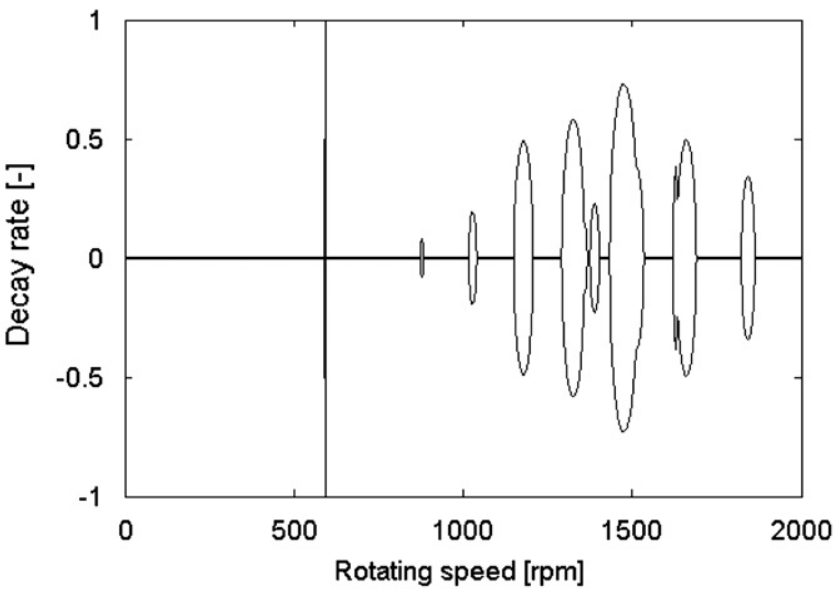

Fig. 14. Stability of the global modes in the general case: (a) in-phase and out-of-phase modes along $x$; (b) decay rate against $\Omega$.

(a)

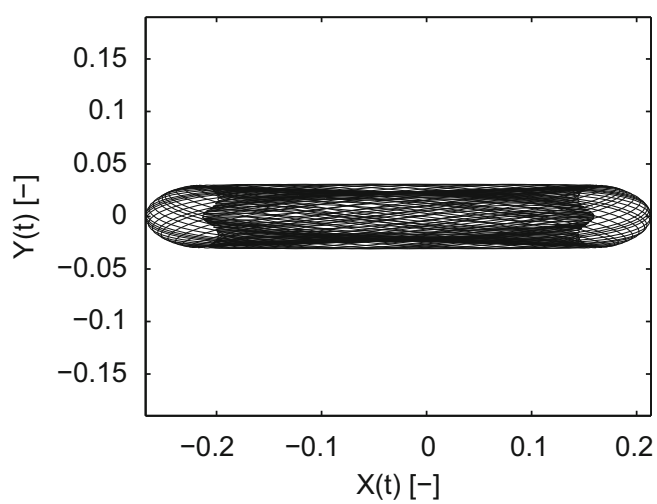

(b)

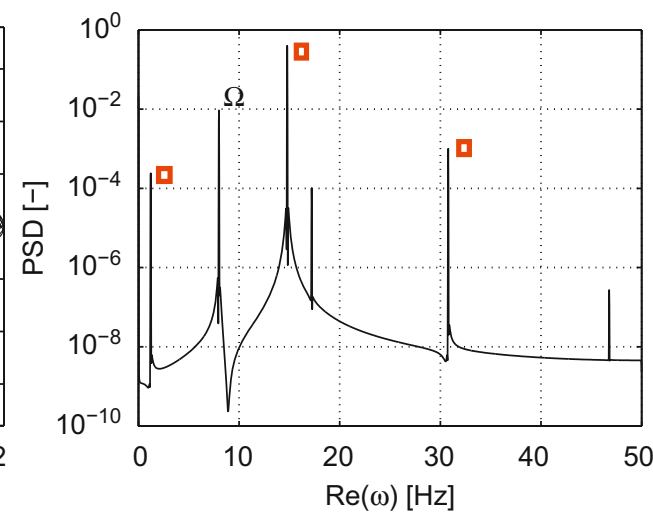

Fig. 15. In-phase parametric quasimodes in $x$ computed for $\Omega=480 \mathrm{rev} / \mathrm{min}$ : (a) orbit of the shaft lower extremity; (b) associated frequency spectrum.

boundary conditions matrix $\mathbf{L}^{j_{\max }}$ given in (32) models the bearings transmission by linking the harmonics of the complex condensed displacements of rotor and stator.

The parametric quasimodes of the rotating test bench are obtained by projecting the eigenproblem (23) on $\boldsymbol{\Phi}_{s s}$ for a given $\Omega$ and a frequential truncature order $j_{\max }$. These simulations lead to four 3D quasimodes deriving from the flexural modes at standstill.

Fig. 15 represents the orbit of a circumferential point of the rotating cylindrical mass for the in-phase quasimode along $x$ computed at $\Omega=8 \mathrm{~Hz}$ and for $j_{\max }=2$. The prevailing mode contribution is directed along $x$ with the fundamental frequency $\omega_{f} \approx 15 \mathrm{~Hz}$ and the secondary harmonics are given by the PSD in Fig. 15(b). The location and magnitude ratio between the harmonic contributions are in good agreement with the experimental results given in Fig. 13(b) comparing the red squares. Because of the truncation of $\mathbf{L}^{j_{\max }}$ to $j_{\max }=2$, the harmonic contributions associated with $j=2$ are obviously not discussed here. Good numerical predictions of the free whirling response are yet reported throughout the range of considered spin speed.

As well, the linear stability of the rotating machine is evaluated through the imaginary part of $\omega$ given in Fig. 14(b). The numerical results highlight several speed range for which occurs the lock-in phenomenon between the harmonics of parametric quasimodes. The small amplitude growth observed experimentally at $\Omega=840 \mathrm{rev} / \mathrm{min}$ appears to be in agreement with the coalescence of the first in-phase quasimode.

\section{Concluding remarks}

Understanding and simulating the global dynamic behaviour of rotating machines are necessary for design and on-line monitoring. Many works have already showed that rotor axisymmetry breaking such as shape imperfections or cracks lead 
to linear periodic time-varying systems governed by Floquet theory. Although lot of developments have been done to simulate the whirling of asymmetric rotor, the suggested finite element models were mostly limited to beam kinematics and, if not, were not taking into account rotor-stator interactions.

This paper introduces the concept of parametric quasimodes which are practical mathematical tools simplifying the understanding of periodic time-varying systems. Their properties have been first investigated through an asymmetric rotating oscillator with two degrees of freedom. In summary, it is always possible to find, in the frequency domain, $n$ independent complex poly-harmonic entities characterizing the Hill governing equations where $n$ is the number of degrees of freedom. Their frequency spectra depend on the periodic coefficient $k(t)$ and the rotational speed $\Omega$ following Poincare's convergence. As for standard eigenmodes, the free whirling and steady-state response are a linear combination of the parametric quasimodes. However, harmonics of the latter are likely to produce lock-in phenomenon, so that the stability of the system is parametric.

These concepts can be extended to the vibration analysis of a complete rotating machine composed of rotating and nonrotating parts modelled in their respective frames. Due to Hill expansion in the frequency domain, the initial degrees of freedom are duplicated leading to a finite set of substructures associated with an harmonic of the Floquet solution. The harmonic coupling due to the axisymmetry breaking is captured by linking the rotating and fixed substructures. This particular modelling conduces naturally to compute the modal basis at standstill with a modal synthesis method. The parametric quasimodes or steady-state response are then obtained by projecting the independent governing equations involving each harmonics on the coupled modal basis.

Finally, some academic experiments are performed to apply and validate the previous concepts. Experimental and numerical results are in very good agreements, suggesting that the numerical method proposed here may be used for modelling every linear time-varying systems requiring 3D kinematics. Indeed, the test bench presented in this paper does not highlight all the possibilities of the finite element method. Damping, gyroscopic coupling, stability and vibratory amplitude response could be investigated right now. For instance, an interesting case in rotordynamics is the vibration analysis of horizontal shafts with breathing cracks [1]. Use of poly-harmonic quasimodes in a 3D finite element method is still valuable but unlike the open crack case, the stiffness is periodic in the rotating frame and its contributions become different for each rotating substructures. The actual difficulty of this method lies in the understanding of the harmonic coupling process to model the effective substructures boundary conditions in 3D kinematics.

\section{Acknowledgements}

The authors wish to acknowledge P. Piteau and T. Valin from the CEA Saclay for their contributions to the experimental investigations.

\section{References}

[1] C.M. Stoisser, S. Audebert, A comprehensive theoretical, numerical and experimental approach for crack detection in power plant rotating machinery, Mechanical Systems and Signal Processing 22 (4) (2008) 818-844.

[2] D. Combescure, A. Lazarus, Refined finite element modelling for the vibration analysis of large rotating machines: application to the gas turbine modular helium reactor power conversion unit, Journal of Sound and Vibration 318 (2008) 1262-1280.

[3] M. Lalanne, G. Ferraris, Rotordynamics Prediction in Engineering, second ed., John Wiley, Chichester, 1994.

[4] G. Genta, Dynamics of Rotating Systems, Springer, New York, 2005.

[5] P.J. Brosens, S.H. Crandall, Whirling of unsymmetrical rotors, Journal of Applied Mechanics 31 (1961) 355-362.

[6] D.J. Han, Vibration analysis of periodically time-varying rotor system with transverse crack, Mechanical Systems and Signal Processing 21 (2007) $2857-2879$

[7] A. Berlioz, J. Der Hagopian, R. Dufour, Dynamic behavior of a drill-string: experimental investigation of lateral instabilities, Transactions of the ASME 118 (1996) 292-298.

[8] G. Floquet, Sur la théorie des equations differentielles (About theory of differential equations), Annales scientifiques de l'ENS 8 (1879) 3-32.

[9] R. Dufour, A. Berlioz, Parametric instability of a beam due to axial excitations and to boundary conditions, Mechanical Systems and Signal Processing 22 (2008) 818-844.

[10] G.W. Hill, On the part of the motion of the lunar perigee which is a function of the mean motions of the sun and moon, Acta Mathematica 8 (1) (1886) $1-36$.

[11] G.M.L. Gladwell, C.W. Stammers, On the stability of an unsymmetrical rigid rotor supported in unsymmetrical bearings, Journal of Sound and Vibration 3 (1966) 221-232.

[12] G. Genta, Whirling of unsymmetrical rotors: a finite element approach based on complex co-ordinates, Journal of Sound and Vibration 124 (1988) $27-53$.

[13] F. Oncescu, A.A. Lakis, G. Ostiguy, Investigation of the stability and steady state response of asymmetric rotors, using finite element formulation, Journal of Sound and Vibration 245 (2001) 303-328.

[14] C.-W. Lee, D.-J. Han, J.-H. Suh, S.-W. Hong, Modal analysis of periodically time-varying linear rotor systems, Journal of Sound and Vibration 303 (2007) 553-574.

[15] A. Nandi, S. Neogy, An efficient scheme for stability analysis of finite element asymmetric rotor models in a rotating frame, Finite Elements in Analysis and Design 41 (2005) 1343-1364.

[16] R. Gasch, A survey of the dynamic behaviour of a simple rotating shaft with a transverse crack, Journal of Sound and Vibration 160 (1993) $313-332$.

[17] H. Poincare, Sur les determinants d'ordre infini (About the infinite determinants), Bulletin de la Société Mathématique de France 14 (1886) 77-90.

[18] C.S. Hsu, On the parametric excitation of a dynamic system having multiple degrees of freedom, Journal of Applied Mechanics (1963) 367-372.

[19] J. Craig, M.C. Bampton, Coupling of substructures for dynamic analysis, AIAA Journal 6 (1968) 1313-1319.

[20] P. Verpeau, T. Charras, A. Millard, Castem 2000: une approche moderne du calcul des structures (Castem 2000: a modern approach to structure computation), Calcul des structures et intelligence artificielle, Pluralis, Paris, France, 1988, pp. 261-271, 〈http://www-cast3m.cea.fr $/\rangle$. 